\title{
Biaxial shear behaviour of HDNR with Mullins effect and deformation-induced anisotropy
}

Laura RAGNI $^{1}$, Enrico TUBALDI ${ }^{2}$, Andrea DALL'ASTA ${ }^{3}$, Hamid AHMADI $^{4}$, Alan MUHR ${ }^{4}$

${ }^{1}$ Department of Construction, Civil Engineering and Architecture, Polytechnic University of Marche, Via Brecce Bianche Ancona (AN), Italy; E-mail: laura.ragni@univpm.it.

${ }^{2}$ Department of Civil and Environmental Engineering, Imperial College London, London, UK; Email:etubaldi@ic.ac.uk

${ }^{3}$ School of Architecture and Design, University of Camerino, Viale della Rimembranza, 63100 Ascoli Piceno (AP), Italy; E-mail: andrea.dallasta@unicam.it

${ }^{4}$ Tun Abdul Razak Research Centre (TARRC), Brickendonbury, Brickendon Lane, Hertford SG13 8NL, UK; E-mail: hahmadi@tarrc.co.uk amuhr@tarrc.co.uk

\section{SUMMARY}

High damping natural rubber (HDNR) bearings, commonly employed to isolate structures, are subjected to biaxial horizontal deformations by the seismic motion. If they have virgin material properties, biaxial models including the stress softening due to the Mullins effect should be used for describing their force-deformation behaviour. Specific studies oriented at characterizing and modelling the biaxial behaviour of HDNR bearings accounting for the stress-softening are very few in number because they would require a large number of virgin isolators or long times of rest between two consecutive tests. Moreover, available studies consider the Mullins effect as an isotropic phenomenon, whereas experimental investigations have shown that it is directiondependent and thus induces anisotropy of the rubber behaviour. This paper describes an experimental campaign carried out on a large number of small material test pieces aimed at achieving a satisfactory characterization of the biaxial anisotropic response of a highly dissipative rubber compound. These results are then used to develop a two-dimensional constitutive model for the virgin HDNR material accounting for the direction-dependence of the Mullins effect, based on the concept of representative directions applied to the biaxial shear deformation state. Because for commonly employed laminated HDNR bearings under design actions the approximation of uniform simple shear is realistic, the proposed material model can also be used to simulate the global bidirectional horizontal response of the bearings.

\section{INTRODUCTION}

Steel-laminated high damping natural rubber (HDNR) bearings show a significant dependence of the horizontal response on the loading history. The shear stress required for loading the rubber layers from the virgin state to a certain strain level is larger than in any subsequent loadings for all strains up to the same maximum strain level. This phenomenon is commonly known as the "Mullins effect" [1] and may be considered as a macroscopic consequence of breakdown of filler-filler structure and rubber-filler interaction which takes place during deformation and mainly depends on the amount of carbon black filler present in the material and the maximum stress applied. Further stress softening also occurs on subsequent cycles to the same strain level, but the stress-strain cycles tend to asymptotically approach stable behaviour. For simplicity, we shall refer interchangeably here to stress softening and the "Mullins effect"; a review of alternative definitions and inconsistencies in the literature was given previously [2]. The stress softening can be induced deliberately on the bearings in production tests (usually termed "scragging") or during earthquakes in service. However, in both the cases it recovers over time. The recovery rate and extent depends 
on rubber formulation details, but has not been studied in much detail. In any case, the virgin horizontal behaviour of bearings should be considered in evaluating the seismic response of isolated structures, given the long time between earthquakes major enough to induce significant stress softening.

Given the multi axial nature of the ground shaking, appropriate models considering the biaxial horizontal behaviour of the bearings and accounting for stress softening should be employed. However, the technical literature on the horizontal biaxial response of seismic isolators [3]-[14] has mainly focused on characterizing the stable (i.e. post-softening) behaviour of the bearings; only three studies [3]-[5] have aimed at quantifying and modelling also the stress-softening. This may be explained by the fact that a large number of virgin isolators or long times of rest between two consecutive tests would be needed for characterizing such strain-history effects. In particular, Huang reported the results of biaxial displacement-controlled tests on an isolated rigid block supported by four laminated HDNR bearings, showing the path and strain-history dependence of the response under different idealised displacement trajectories [3]. This model was later extended and improved by explicitly including the stress softening [4]-[5]. In particular, two different degradation mechanisms have been adopted by Grant et al. [5], one for simulating the "scragging" and the other for simulating the "Mullins effect", defined in [6] to be first-cycle and continuous cyclic softening respectively. However, the first contribution (which is the most important and accounts for the dependence on the maximum experienced strain) does not evolve for any strain history, because it does not fully take effect until the bearing is unloaded. Moreover, while the parameters relevant to the "Mullins effect" have been calibrated based on several tests (separated by time periods ranging from 5 minutes to 24 hours), the model for the "scragging" has been calibrated based on just one test, carried out on a virgin device. This model was recently included in a three-dimensional bearing model developed by Kumar et al. [7] and implemented in Opensees [8], accounting for other specific phenomena such as the cavitation/buckling phenomena occurring under tensile/compression strains [9] and the interaction between the horizontal and vertical behaviours. The other studies in the technical literature on multi-axial testing and modelling of HDNR isolators do not consider stress softening [10]-[14], although it was observed in the bearing response.

More experimental and modelling information about the Mullins effect can be found in papers dealing with the material behaviour of HDNR, because it is less costly to study the virgin stressstrain behaviour of material test pieces rather than that of full sized bearings. Material models accounting for the Mullins effect have been developed, based for example on coupling of continuum damage theory with elasticity theory [15], pseudo-elasticity theory [16], or viscoelasticity theory [17]. However, in most cases the Mullins effect is modelled as an isotropic phenomenon, whereas experimental investigations have demonstrated that it is direction-dependent. In particular, Besdo et al. [18] and Diercks and Lion [19], based on simple shear strain test of filled rubber under different directions, have shown that while a loading in one direction results in a reduced stress for subsequent loading in the same direction (stress-softening), the effect is less pronounced in other directions. The same conclusion is reached by Dorfmann and Pancheri [20] by performing a series of uniaxial and planar biaxial tension tests. Physical explanations for such deformation-induced anisotropy have been proposed, e.g. in [21], and some micro-mechanical material models, such as a three-dimensional one by Göktepe and Miehe [22] have been developed. Less complex phenomenologically-based approaches have also been employed to model deformation-induced anisotropy, such as the constitutive model based on the pseudo-elasticity theory and accounting for the Mullins effect and the changes in the material symmetry proposed by Dorfmann and Pancheri [20]. In the same paper an interesting overview of other available phenomenological models is given. Among these, the concept of representative directions [23] appears very convenient, since it allows to generalize the one-dimensional material laws to describe three-dimensional material behaviour.

This paper aims to extend the uniaxial model developed in [2] for the non-linear, hysteretic and history-dependent stress-strain behaviour of virgin HDNR material and accounting for the stress- 
softening to cover general biaxial shear states typical of rubber bearings. Under the assumption of uniform simple shear strain in the rubber layers of laminated HDNR bearings, realistic for commonly employed shape factors of bearings and design values of axial load [24]-[25], the proposed material model can be used to simulate the global bidirectional horizontal response of virgin HDNR bearings, by means of simple geometrical considerations.

The paper first describes an extended experimental campaign carried out on double and single shear specimens, manufactured by TARRC from a HDNR compound usually employed for seismic isolators and showing a significant stress softening similarly to other highly dissipative rubber compounds [26]. These tests have been devised to better understand and hence model the deformation-induced anisotropy due to the Mullins effect, as well as the coupled bi-axial shear behaviour of the HDNR material. The use of material test pieces rather than bearings has enabled a large number of tests on separate virgin HDNR samples to be performed, so as to achieve a satisfactory characterization of stress softening. In the second part of the paper, a phenomenological model for the HDNR material under biaxial shear accounting for stress-softening is proposed, whose parameters are calibrated against the experimental results. The stress-softening of the proposed model provides a satisfactory description of the transient response of the rubber (characterizing the transition from the virgin state to the damage stabilization) and it is based on a degradation model taking into account, for any strain history, the dependence on the maximum strain experienced and the effects of the deformation-induced anisotropy. In particular, the use of the representative directions approach is employed to generalize uniaxial shear models, previously developed by some of the authors of this study [2],[26]-[29], to a bi-axial shear model for HDNR.

\section{EXPERIMENTAL CAMPAIGN}

This section illustrates the experimental campaign aimed at studying the direction-dependence of the Mullins effect and the resulting deformation-induced anisotropy of the transient response of filled rubber. Small material test pieces have been employed, due to the large number of independent tests that have to be carried out on separate virgin HDNR samples to achieve a satisfactory characterization of the stress softening affecting the transient response. All the rubber specimens have been manufactured by TARRC using a rubber compound employed for seismic isolators and satisfying the prescriptions of the current European code for anti-seismic devices [25] about the stability of shear properties under repeated cycling (the ratio between the stiffness of the tenth and first cycle must not be less than 0.6). The results of uni-axial double shear tests, aimed at characterizing the virgin and stable one-dimensional behaviour of this rubber, are first reported. Since the focus of the paper is on the bi-axial behaviour of virgin filled rubbers, only some results are included and a more complete description of these tests may be found in [2]. The second set of tests consists of rotated uni-axial double shear tests, in which the testpieces are first loaded in one direction, then rotated, and finally reloaded in different directions. This enables the dependence of the Mullins effect on the reloading direction to be investigated. Finally, biaxial tests are carried out to study and characterize the virgin and stable rubber behaviour under bi-axial shear, with particular attention to the deformation-induced anisotropy and coupling effects between deformations on the two axes.

\section{Uni-axial double shear tests}

The testpiece for double-shear tests consists of two cylindrical rubber discs (Figure 1a) moulded between three metal end pieces. The nominal thickness of the disc is $6 \mathrm{~mm}$ and its diameter is $25 \mathrm{~mm}$, complying with Standards for testing rubber in simple shear [30] which prescribe a ratio of width (in the direction of shear) to rubber thickness $>4$ to justify the assumption that the rubber is in uniform simple shear. The two external metal end pieces are fixed in the stationary clamp of the testing machine (Figure 1b), attached to the load cell. For each test the load cell force and the imposed displacements are recorded by the testing machine's internal sensors. Results are reported 
in terms of shear stress $(\tau)$ versus shear strain $(\gamma)$, calculated by dividing the measured force and displacement by the area and the thickness of the testpiece respectively.

Double shear tests have been carried out at different strain amplitudes and strain rates. Since the dependence on the strain rate is quite low [2], only the results of tests carried out with a strain rate equal to $\dot{\gamma}=4 \mathrm{~s}^{-1}$ are reported in this paper. Figure $2 \mathrm{a}-\mathrm{d}$ illustrates the shear stress-strain cycles of four different virgin rubber test pieces subjected to maximum strain amplitudes $\gamma_{\max }$ equal to 2, 1.5, 1 , and 0.5 . The strain path of each test consists of 20 cycles at the maximum strain amplitude followed by a further 20 cycles at each smaller amplitude. For example, in Figure 2 the 20 cycles at $\gamma_{\max }=2$ are followed by 20 cycles at amplitudes equal to $1.5,1,0.5$ and 0.25 .

a)

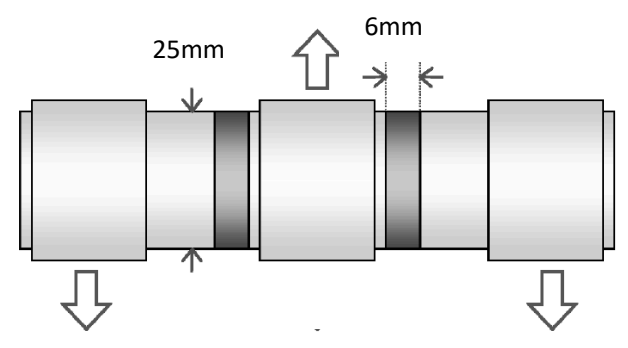

b)

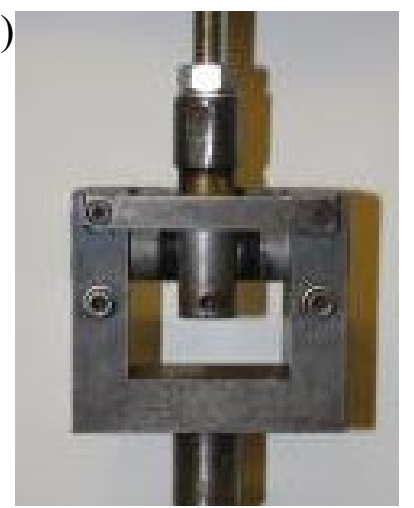

Figure 1. Test set-up: (a) double shear test piece geometry and (b) test machine

a)
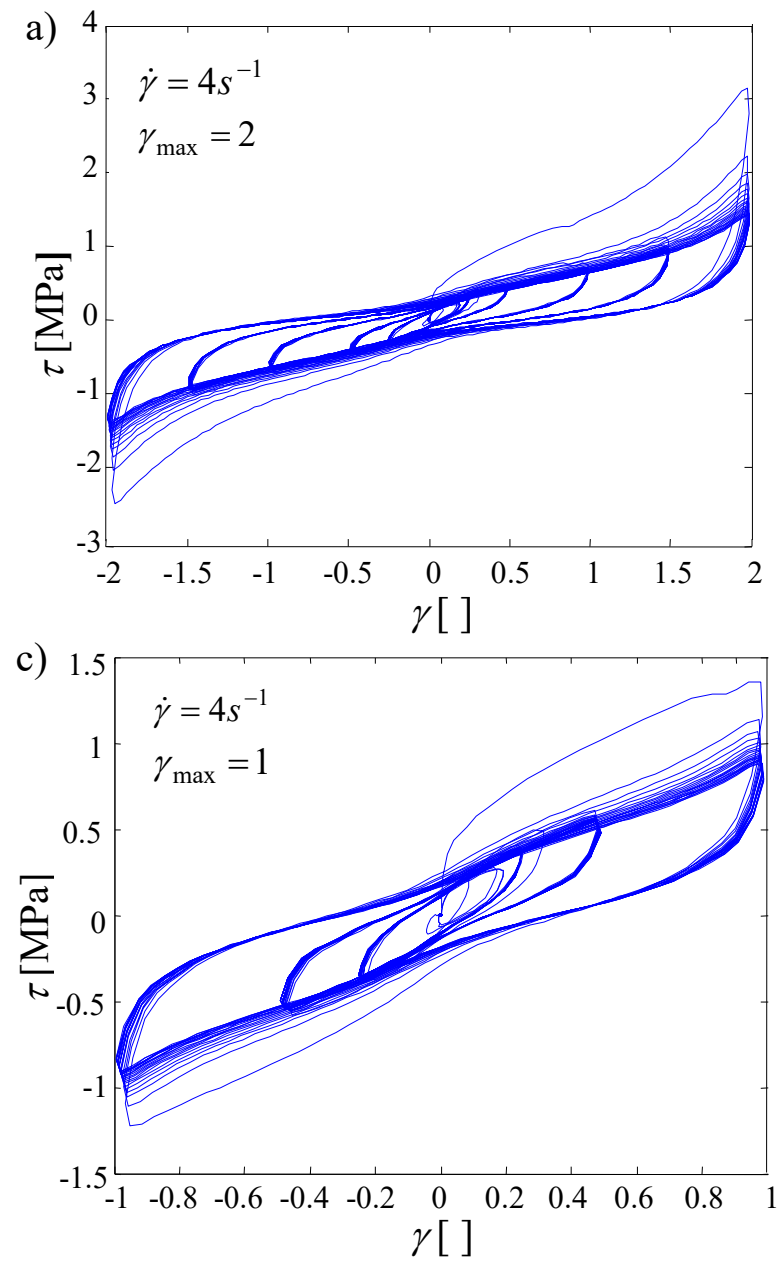

b) 2.5
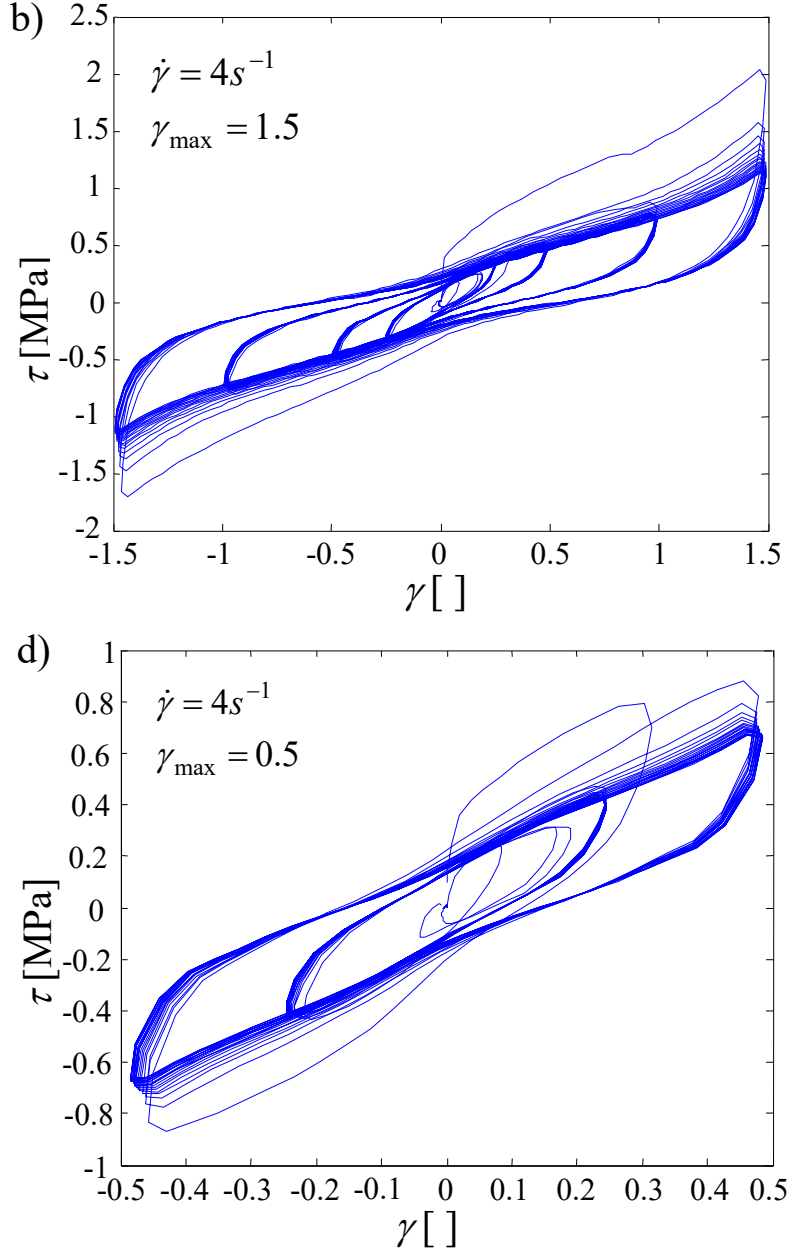

Figure 2. Uniaxial shear test results at strain rate $4 \mathrm{~s}^{-1}$ corresponding to maximum shear strains of (a) 2.0, (b) 1.5, (c) 1.0 and (d) 0.5 . 
It is evident from the figures that the stress-softening characterizing the shear response under repeated cycling is significant, and that, once the stress-softening is stabilized for the cycles at the maximum strain amplitude, successive cycles at smaller amplitudes are also stable (no additional stress softening occurs) and their stiffness increases as the strain amplitude decreases (Payne effect). In order to show the dependence of the stress-softening level on the maximum deformation previously experienced by the rubber, only the stable cycles observed at the maximum strain amplitude for each test are reported in Figure 3a. It is evident that the stiffness associated with a cycle is the higher, the lower is the maximum previously experienced strain, thus resulting in less stress-softening of the rubber. This is also confirmed by the comparison reported in Figure $3 \mathrm{~b}$, reporting for all the tests the stable cycles at the strain amplitude equal to 0.5. It is evident that the stiffness of these cycles decreases as the maximum strain amplitude previously imposed increases.

Beside the dependence on the maximum experienced strain, another important aspect of the Mullins effect that can be analyzed by means of uni-axial double-shear tests, is the dependence on the strain direction (positive and negative strains), which has been reported also in other experimental investigations [18]-[19]. To this end, a second series of tests have been carried out, in which a pair of virgin rubber samples have been subjected to the strain histories reported in Figures 4a. The first strain history consists of a common sequence of 6 cycles with triangular shape at an amplitude of 1.5 and strain rate of $2 \mathrm{~s}^{-1}$, whereas the second one consists of 6 half cycles with triangular shape at an amplitude of 1.5 followed by 6 half cycles with triangular shape at an amplitude of -1.5 . Figure $4 \mathrm{~b}$ shows the hysteretic cycles corresponding to the applied strain histories. The comparison between the results of the two tests clearly shows that the stress-softening occurring in one direction has a negligible influence on the stress-softening in the other direction.
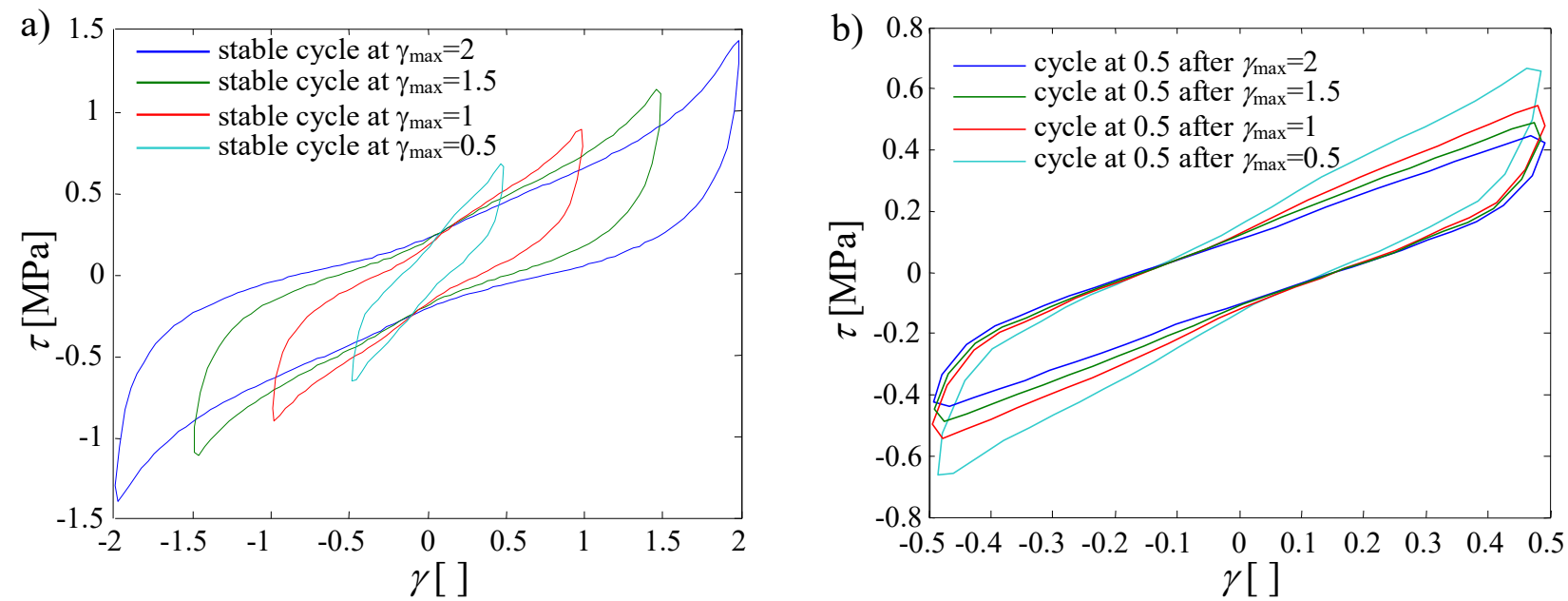

Figure 3. Stable cycles for uniaxial shear test results: (a) at different maximum strain amplitudes and (b) at a strain amplitude of 0.5 after different maximum strain amplitudes
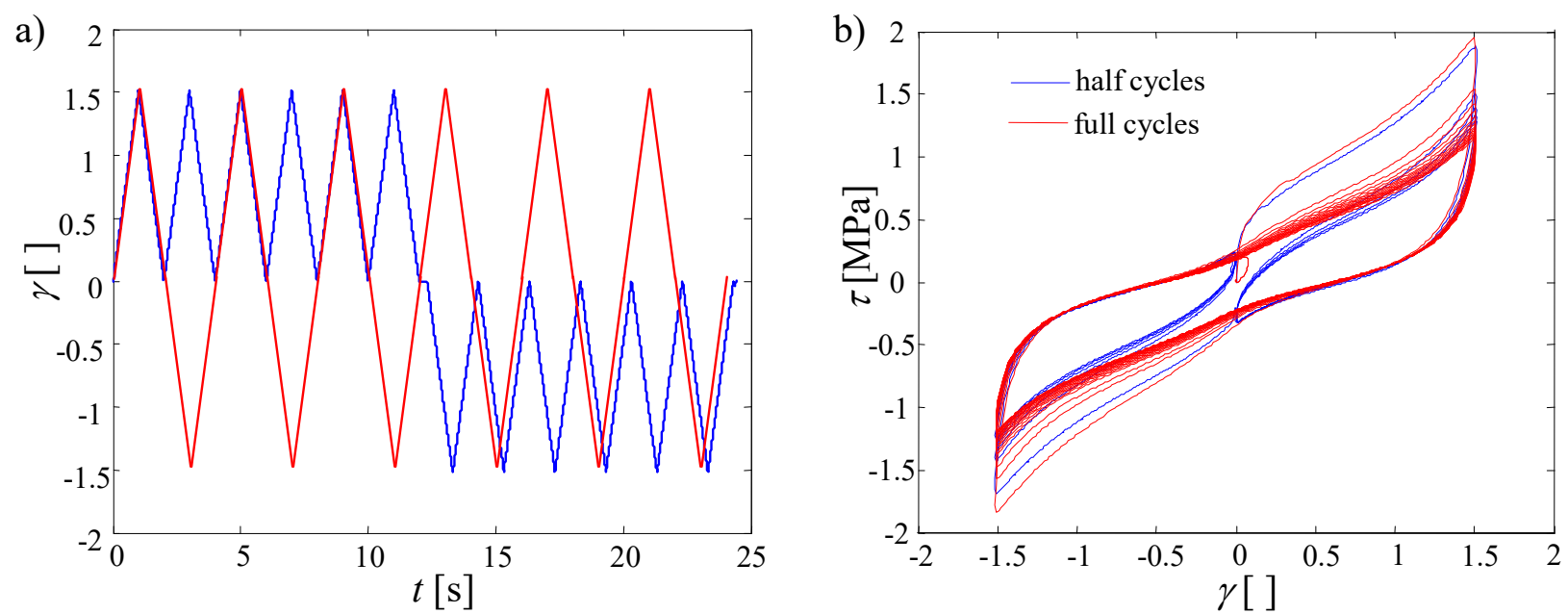

Figure 4. Symmetric and asymmetric tests: (a) strain histories imposed and (b) hysteretic response. 


\section{Rotated uni-axial double shear tests}

This section illustrates a series of experimental tests conducted on many different vulcanized virgin material samples in simple-shear under strain histories acting in different directions.

The cylindrical double shear testpieces were installed in a special jig in a uniaxial servohydraulic testing machine. This jig permitted rotation of the testpiece about its axis, such that all three metal pieces go through the same angle so as not to induce torsion between consecutive uniaxial tests along the invariant machine axis. During these uniaxial tests, the metal pieces were firmly clamped to eliminate any backlash or tendency to rotate. Starting from zero shear strain, the special jig enabled the testpieces to be subjected to a sequence of cycles in uniaxial shear, first in the reference direction (denoted by a rotation angle $\theta=0^{\circ}$ ), then in a different direction rotated of an angle $\theta$ with respect the reference one. In particular, each virgin rubber specimen has been subjected to 6 consecutive cycles in a sequence of increasing strain amplitudes along each of two different directions: the reference direction (first) and the rotated direction (after the six cycles along the reference direction). The strain amplitudes considered for the cycles are $0.25,0.5,1,1.5$, and 2.5 (with a strain rate equal to $2 \mathrm{~s}^{-1}$ ) whereas the adopted values of $\theta$ are: $0^{\circ}, 30^{\circ}, 60^{\circ}, 90^{\circ}, 135^{\circ}, 180^{\circ}$. In order to allow time to rotate the specimen, 60 seconds of rest between cycling at every amplitude and direction $0^{\circ}$ have been imposed, as shown in Figure 5. Figure 6 shows the stress-strain loops obtained. The response without rotation $\left(0^{\circ}\right.$ of rotation) is illustrated in Figure $6 \mathrm{a}$. It is important to note that the contribution of the Mullins effect is strongly reduced when repeating the tests in the same direction, though it is not negligible due to some recovery occurring between the time intervals of rest of the test sequence. However, from the Figures $6 \mathrm{~b}-6 \mathrm{f}$ it is evident that an additional softening, in the new direction, becomes more and more pronounced in the repeated test (red) as the rotation angle increases.

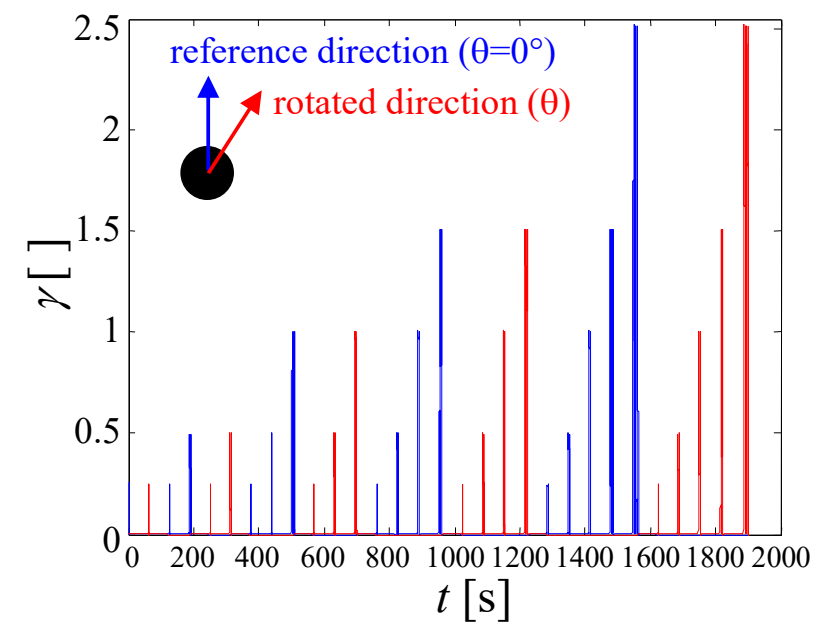

Figure 5. Imposed strain history of the rotated double shear test.
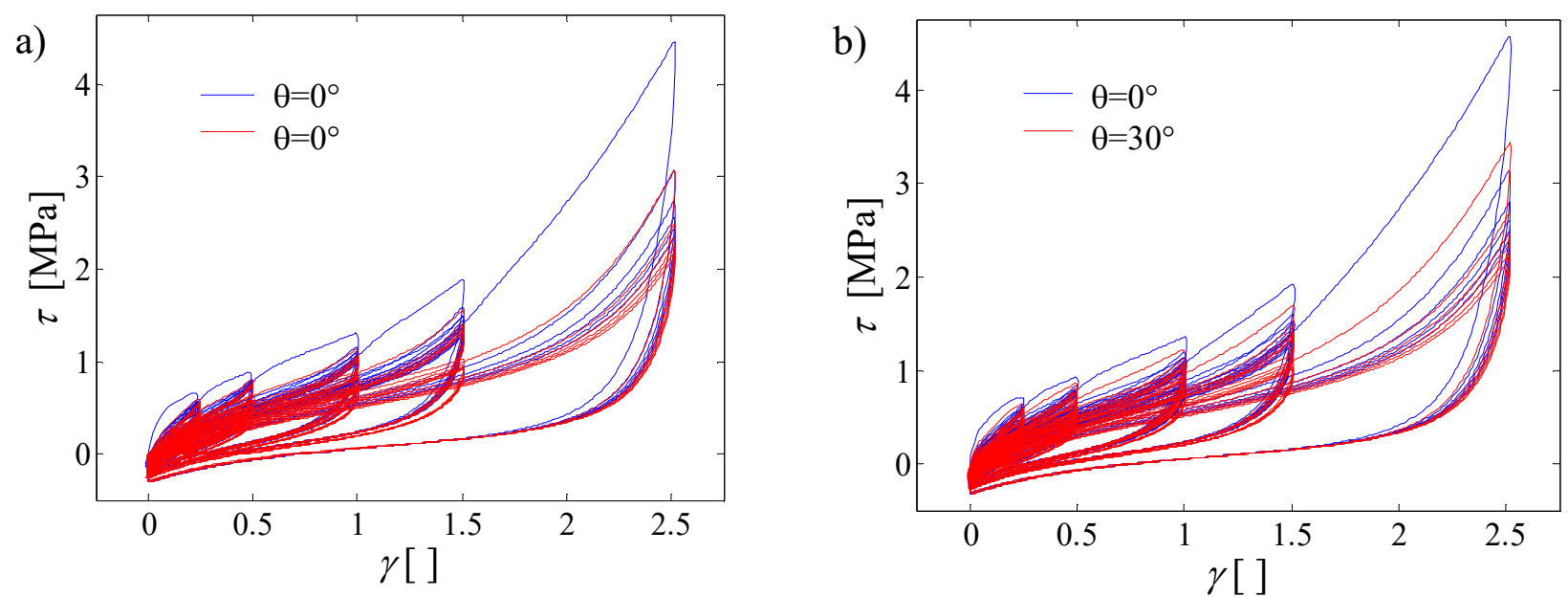

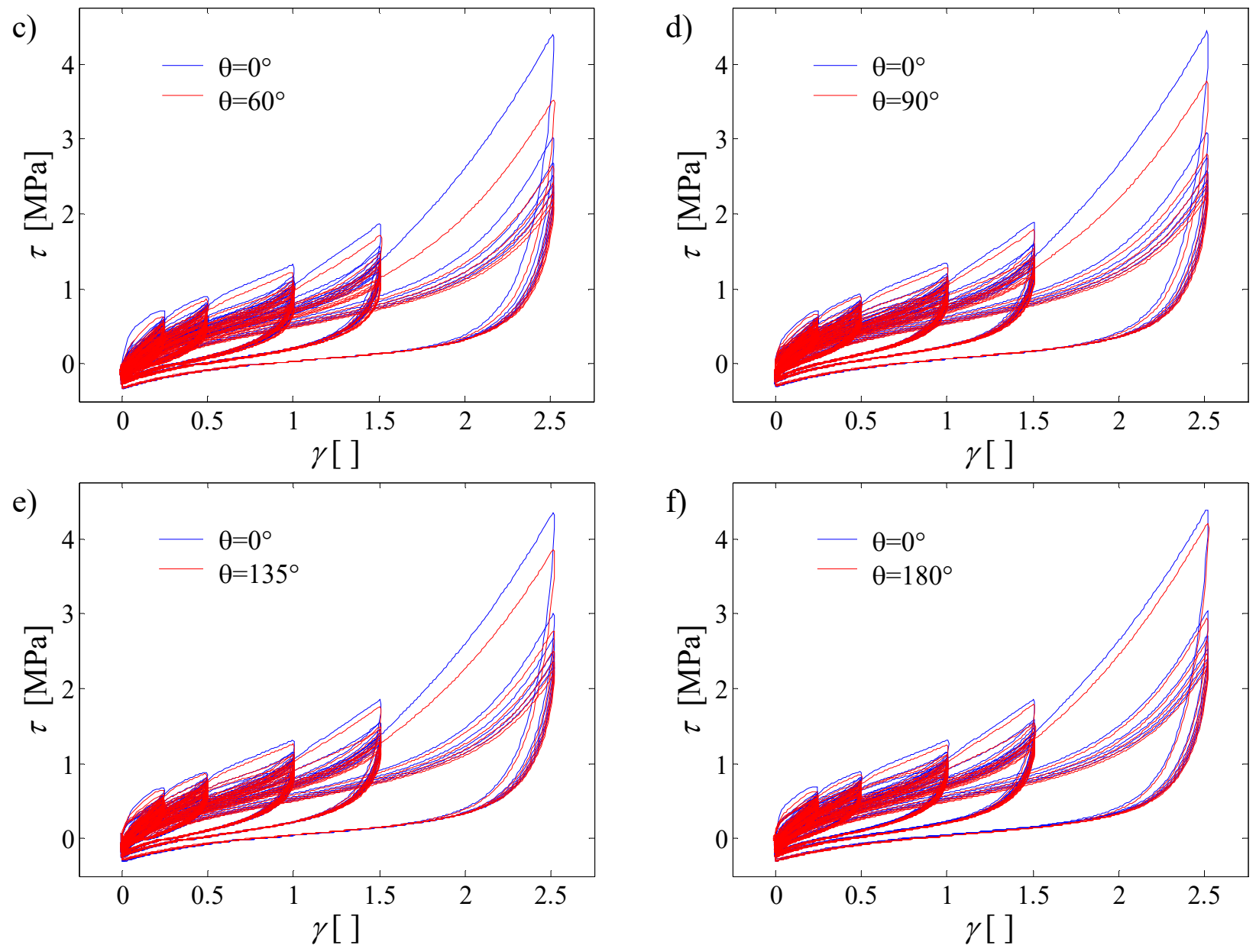

Figure 6. Double shear tests on rotated testpieces: (a) $\theta=0^{\circ}$, (b) $\theta=30^{\circ}$, (c) $\theta=60^{\circ}$, (d) $90^{\circ}$, (e) $\theta=135^{\circ}$ and (f) $\theta=180^{\circ}$.

Finally, Figure $6 \mathrm{f}$ shows that the response in the direction with $180^{\circ}$ of rotation is very close to the response in the reference direction of the virgin testpiece, which confirms that the Mullins effect depends on the sign of the strain, consistent with the uni-axial test results described previously. In other words, the softening effect of the half cycles in the reference direction on the subsequent behaviour in the opposite direction is nearly negligible; for intermediate angles, the softening effect lies in between these limits.

\section{Bi-axial shear tests}

The last part of the experimental campaign consisted of bi-axial shear tests performed on separate virgin HDNR samples, with particular attention to the deformation-induced anisotropy characterizing the transient response due to the direction-dependence of the Mullins effect, to elucidate the response of the virgin rubber under biaxial inputs. Different strain trajectories were used in the tests, to probe both the Mullins and the biaxial coupling effects in a comprehensive but also a systematic way. These trajectories have been controlled by imposing a biaxial (but not torsional) relative motion to the two steel plates bonded to the rubber specimen, which consisted of a cylindrical single rubber layer of thickness $h$ equal to about $8 \mathrm{~mm}$ and radius $a$ equal to $25 \mathrm{~mm}$ (Figure 7).

Parallel motion of the metal plates between which the rubber layer is bonded essentially imposes a state of nominal uniform simple shear on the rubber provided that the width to height ratio of the rubber specimen is sufficiently high, because the imperfection in the boundary conditions due to the stress-free edges of the rubber then becomes less significant. Standards for testing rubber in simple shear [30] stipulate a minimum value of this ratio equal to 4 , while the value for the tested pieces is 6.25. The biaxial horizontal displacements have been imposed on the specimens, by means of two 
plates sliding on horizontal orthogonal linear bearings. Each plate was attached to a horizontal actuator, with maximum load and stroke capacities respectively of $10 \mathrm{kN}$ and $150 \mathrm{~mm}$. Here again, results are reported in terms of shear stress $(\tau)$ versus shear strain $(\gamma)$, calculated by dividing the measured force and displacement by the area and the thickness of the testpiece respectively. In addition to the horizontal displacement, a sliding plate on a vertical linear bearing was used to impose a vertical deformation, producing an initial vertical pressure of $2 \mathrm{MPa}$.

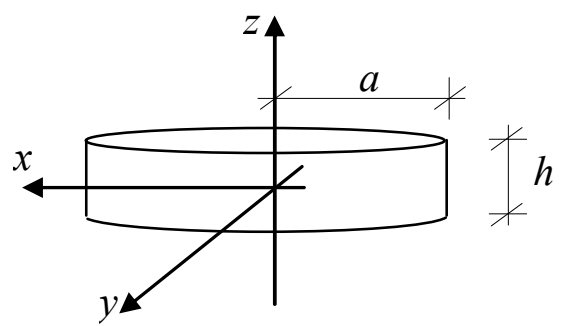

Figure 7. Single rubber layer bonded between shims

Figure 8 shows the test rig used for performing the test. Throughout the experiments, the displacements and forces in the horizontal directions have been measured by internal sensors of the actuator. Forces, free of the rolling resistance of the linear bearings, have also been measured by a triaxial load cell connecting the testpiece to the lower linear bearing. In general, the differences observed between the forces measured by the two instruments were very low. Thus the forces measured by the internal sensor of the actuators are reported hereinafter.

a)
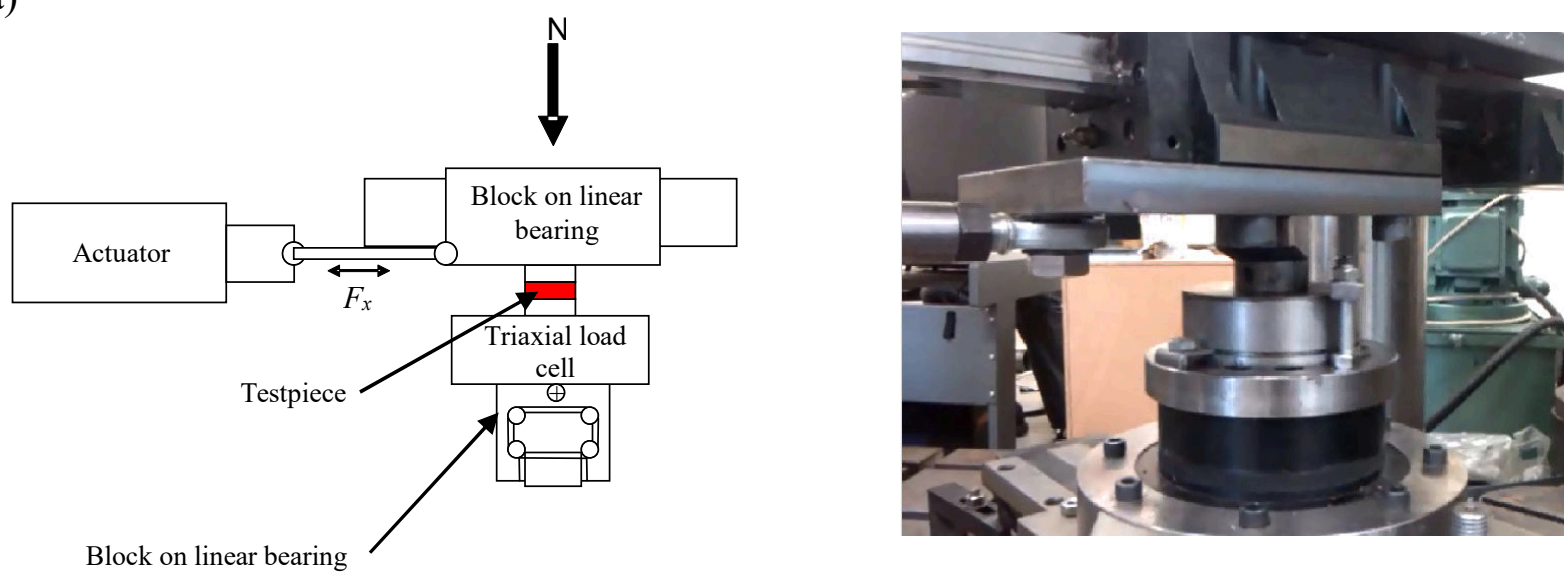

b)
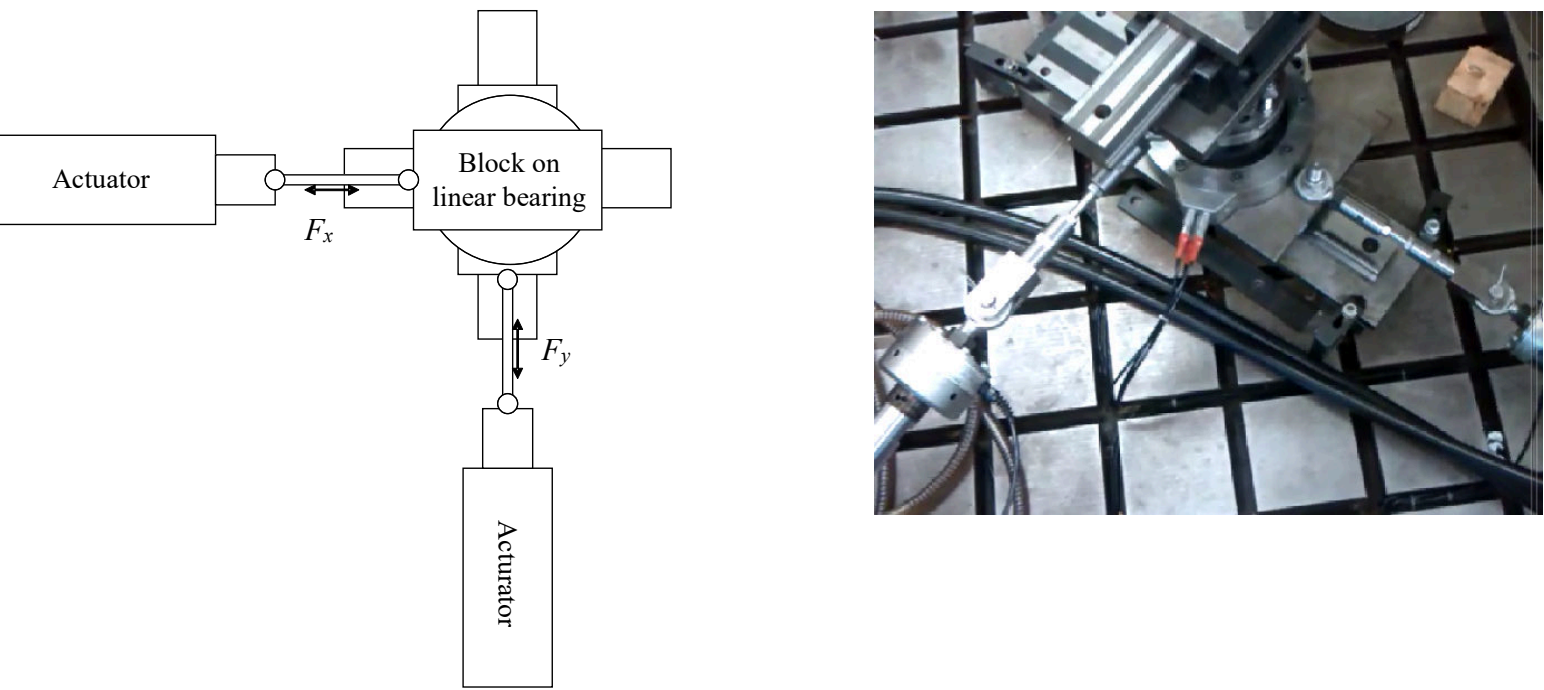

Figure 8. Biaxial test rig: (a) front view and (b) plan view (b). 
A series of cyclic two-directional displacement trajectories of different shapes and amplitudes have been imposed on the rubber layers. The tests corresponding to a cruciform trajectory in the strain plane are reported first. These tests consist of imposing a cyclic sinusoidal displacements in one direction $(y)$ and then in the orthogonal direction $(x)$ as shown in Figure 9a. The frequency of the imposed motion is $0.5 \mathrm{~Hz}$ and the amplitude $\gamma_{\max }$ is 1.5 , for consistency with frequency and amplitude of laminated rubber bearings usually employed for seismic isolation. The stress trajectory as well as the stress-strain diagrams along the $x$ and $y$ directions are reported in Figures 9b-9d. This test confirms that cycling in one direction $(y)$ up to the stable behaviour has a not negligible effect on the response along the orthogonal direction $(x)$. However, the amount of stress-softening along $x$, although not negligible, is reduced with respect to that along $y$, showing the Mullins effect induces anisotropy. Results of this bi-axial test along the first load direction $(y)$ are compared in Figure $9 \mathrm{~d}$ with the results of the uni-axial test carried out on double-shear specimens at the same maximum strain amplitude (red dashed cycles). The two plots are very similar, confirming that the vertical load has negligible influence.
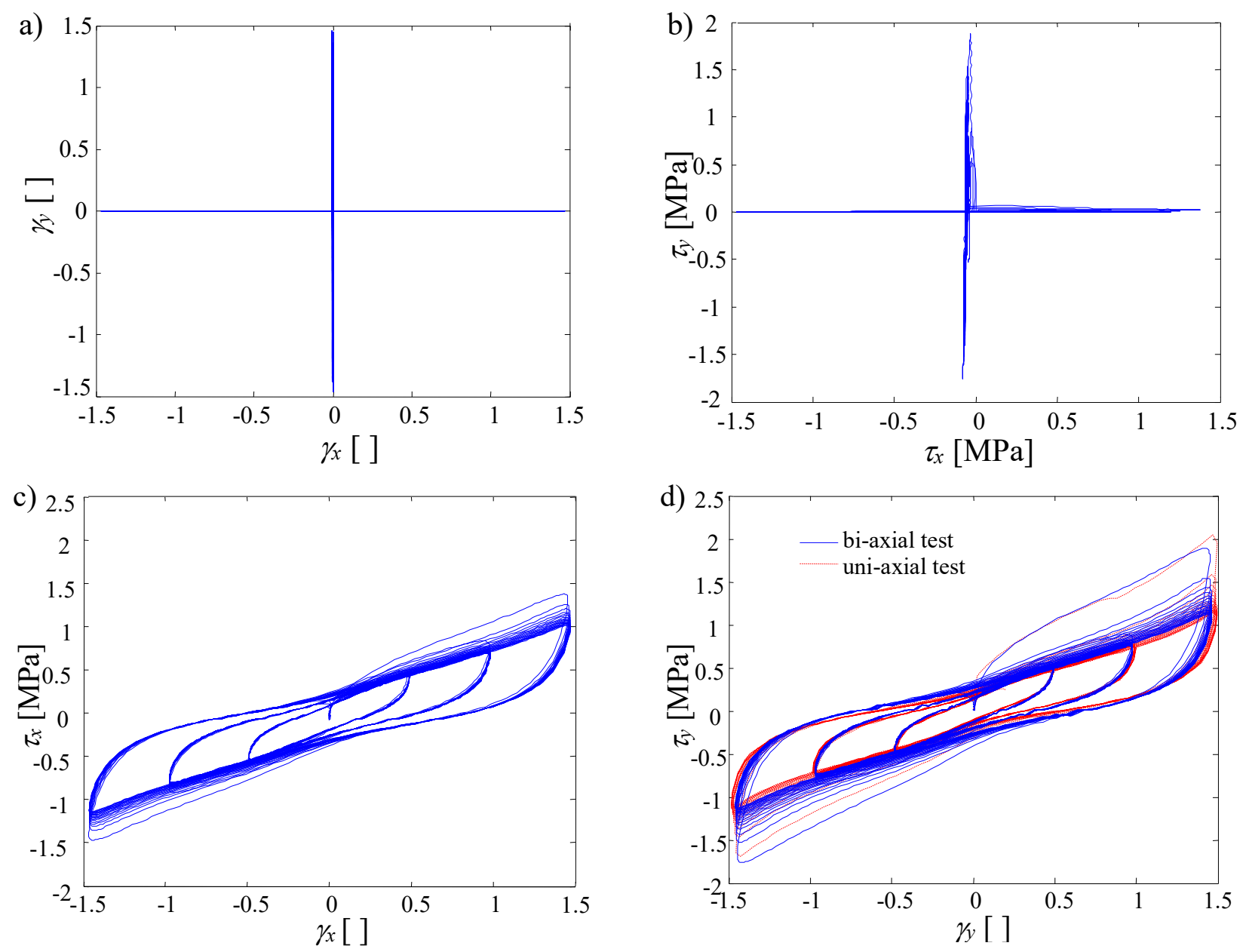

Figure 9. Biaxial tests: (a) cruciform strain trajectory at 150\%, (b) stress trajectory, (c) stress-strain response in the $x$ direction, (d) stress-strain response in the $y$-direction

Circular strain trajectories have also been imposed to study the consequence of the directiondependence of the Mullins effect on the bi-axial rubber response. A circular trajectory of radius $\gamma_{\max }$ $=1.5$ in the shear strain plane was first applied. This trajectory has been obtained by imposing, after a ramp in the $x$-direction up to $\gamma_{\max }$, sinusoidal motions at the same frequency of $0.5 \mathrm{~Hz}$ in the two orthogonal directions with a phase lag of $\pi / 2$. Circular trajectories at smaller strain amplitudes $\left(\gamma_{\max }\right.$ $=1.0$ and $\left.\gamma_{\max }=0.5\right)$ have also been imposed after the larger circular trajectory; 20 cycles were applied at each amplitude. Figure 10 reports the stress trajectory and the stress-strain loops. The 
stress trajectory reported in Figure $10 \mathrm{~b}$ shows that after the application of the ramp along the $x$ direction, a relaxation process occurs, with the maximum stress falling from about $1.9 \mathrm{MPa}$ (point $\mathrm{A}$ of Figure 10b) to about 1.5 MPa (point B of Figure 10b). Assuming that this effect has vanished after one half cycle of deformation, as in the previous tests, the maximum stresses observed in the $x$ and $-y$ directions (points $\mathrm{C}$ and $\mathrm{D}$ of Figure $10 \mathrm{~b}$ ) are similar to the maximum value of $1.9 \mathrm{MPa}$ observed in the $+x$ direction (point A of Figure 10b). This confirms that a softening of the material in one direction has little influence on the behaviour in other directions. Finally, it is important to observe that during all the response history the stress resultant is not oriented as the strain vector. In fact, when the stresses are at points $\mathrm{C}$ and $\mathrm{D}$ in the stress trajectory, the strains are at points $\mathrm{C}^{\prime}$ and $\mathrm{D}^{\prime}$ in the strain trajectory. In the case of linear elastic behaviour, the orientation of the stresses and of the strains would be the same. Figures $10 \mathrm{c}$ and $10 \mathrm{~d}$ report the shear stress-strain diagram in the two orthogonal directions ( $x$ and $y$ ). Also in this case, the results in terms of stress-strain diagrams are compared with the results of the uni-axial double shear tests carried out at the same maximum strain amplitude (red dashed cycles in Figure 10c and 10d). As expected, the two initial load paths, in the $+x$ direction of Figure 10c, are very similar whereas the stable cycles are significantly different. This shows that the stress in one axis is not only influenced by the deformation in that axis, but also by the simultaneous deformation in the orthogonal axis: i.e. there is a marked coupling effect. The coupled horizontal behaviour results for this trajectory in a larger hysteresis loop than in the case of uncoupled uni-axial behaviours, as also observed in [10]. The circular test has been repeated on a different testpiece with a maximum amplitude $\gamma_{\max }=1.0$ and similar conclusions may be drawn for the test results (Figure 11).
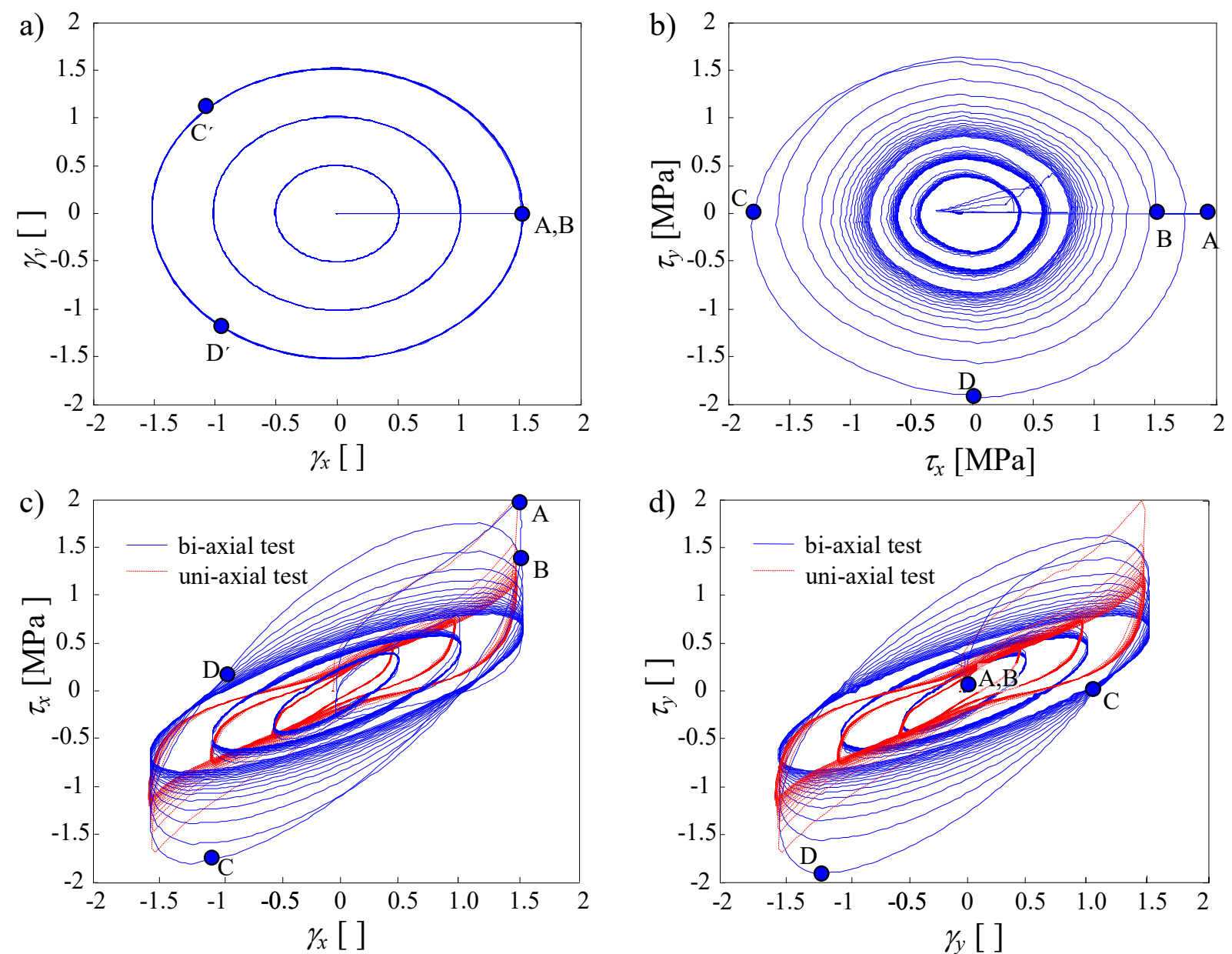

Figure 10. Biaxial tests: (a) circular strain trajectory at 150\%, (b) stress trajectory, (c) stress-strain response in the $x$ direction, (d) stress-strain response in the $y$-direction. 

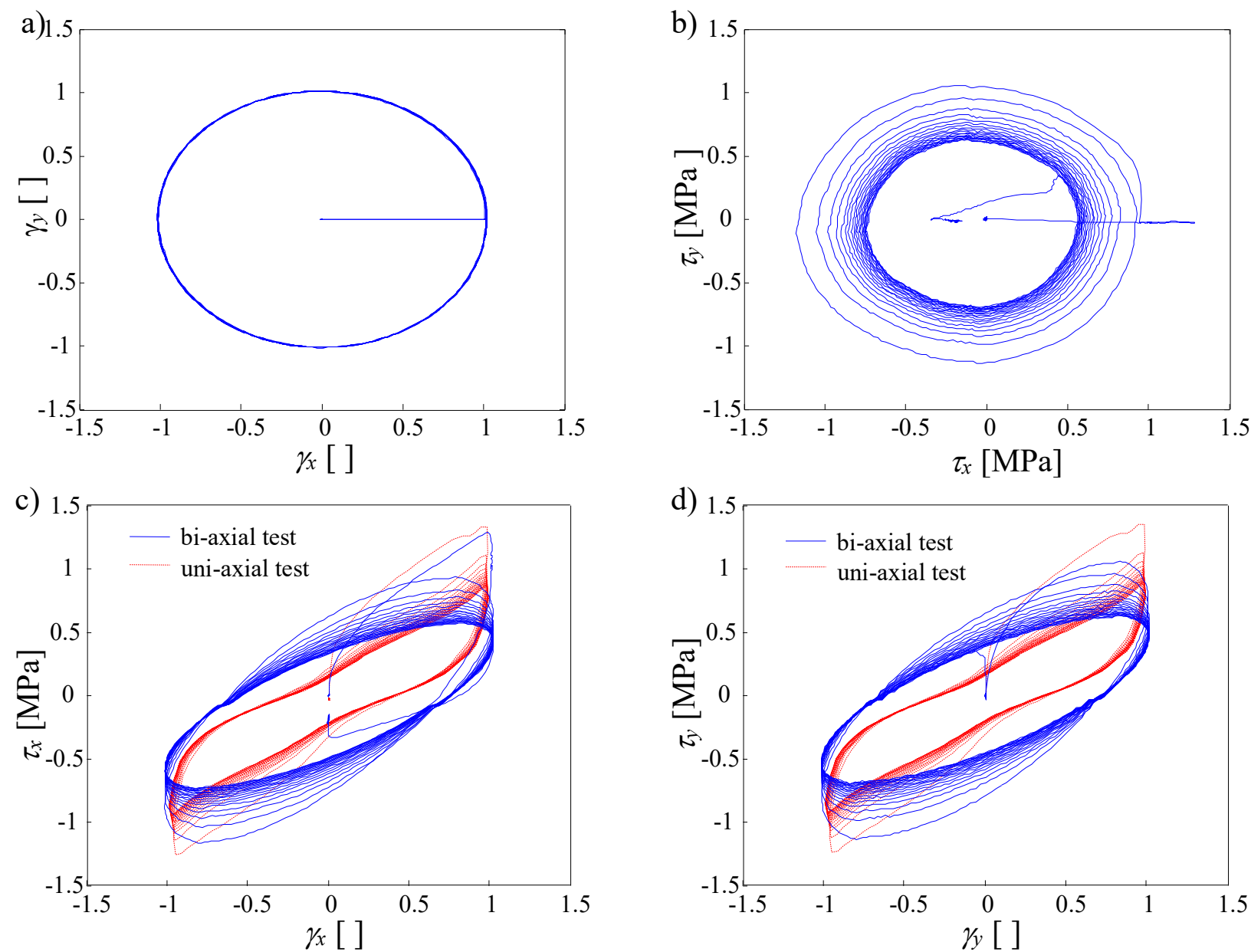

Fig 11. Biaxial tests: (a) circular strain trajectory at 100\%, (b) stress trajectory, (c) stress-strain response in the $x$ direction, (d) stress-strain response in the $y$-direction

A square trajectory (Figure 12 a) and a figure 8-shaped trajectory (Figure 13a) have also been carried out on other virgin specimens at a maximum strain amplitude level of 1.0. The figure 8shaped trajectory was obtained by imposing a sinusoidal displacement at a frequency of $0.5 \mathrm{~Hz}$ along the $y$-direction and at twice this frequency along the $x$-direction. The test with the square trajectory is particularly interesting because it confirms the deformation-induced anisotropy due to the Mullins effect, since similar observations about the first cycle may be made as for the circular trajectory case. Moreover, by comparing the strain and stress trajectories reported in Figure 12a and $12 \mathrm{~b}$, a rotation of the stress resultant with respect to the imposed displacement can be observed, which provides an indirect measure of the dissipation properties of the material.
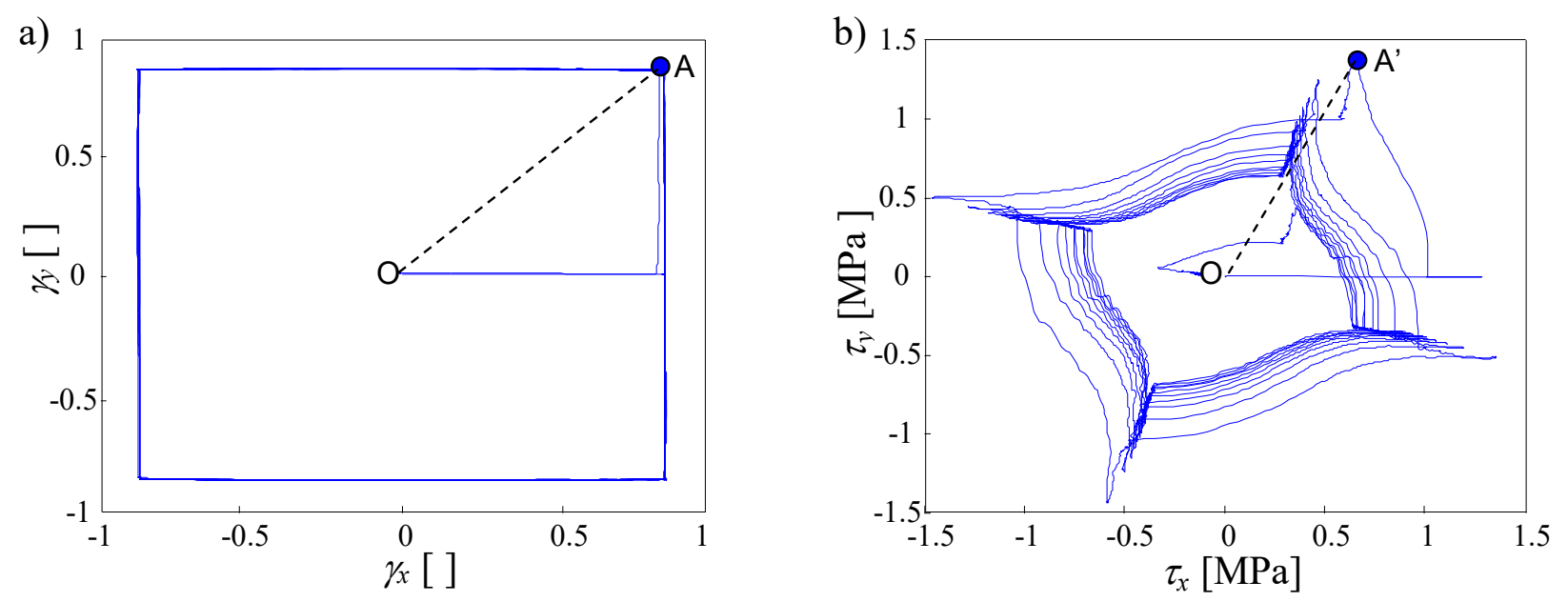

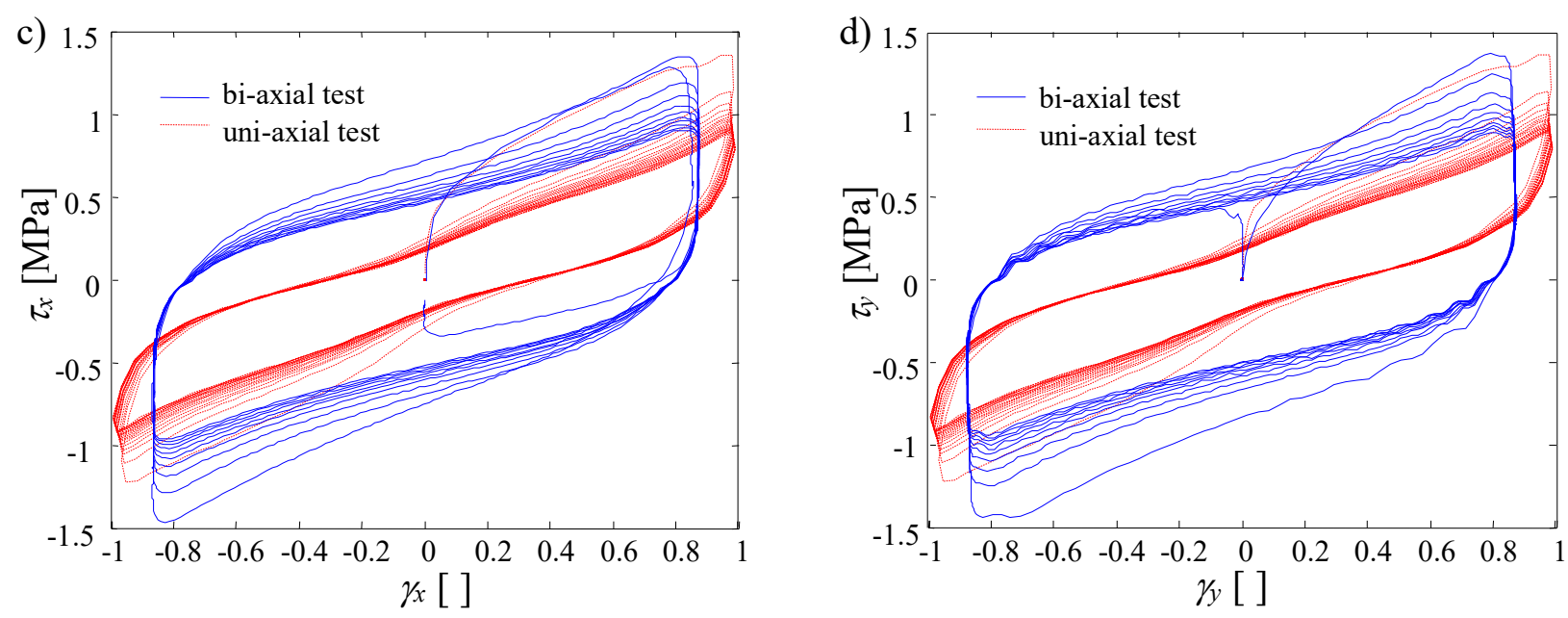

Figure 12. Biaxial tests: (a) square strain trajectory at 100\%, (b) stress trajectory, (c) stress-strain response in the $x$ direction, (d) stress-strain response in the $y$-direction

In fact, if the material behaviour were isotropic elastic, the stress would be oriented as the strain, and the stress trajectory would not be rotated (segments OA and OA' in Figure 12 would have the same direction). Thus, the rotation angle between these two segments is due to dissipation mechanisms of the material and this can be useful for calibrating the material response.
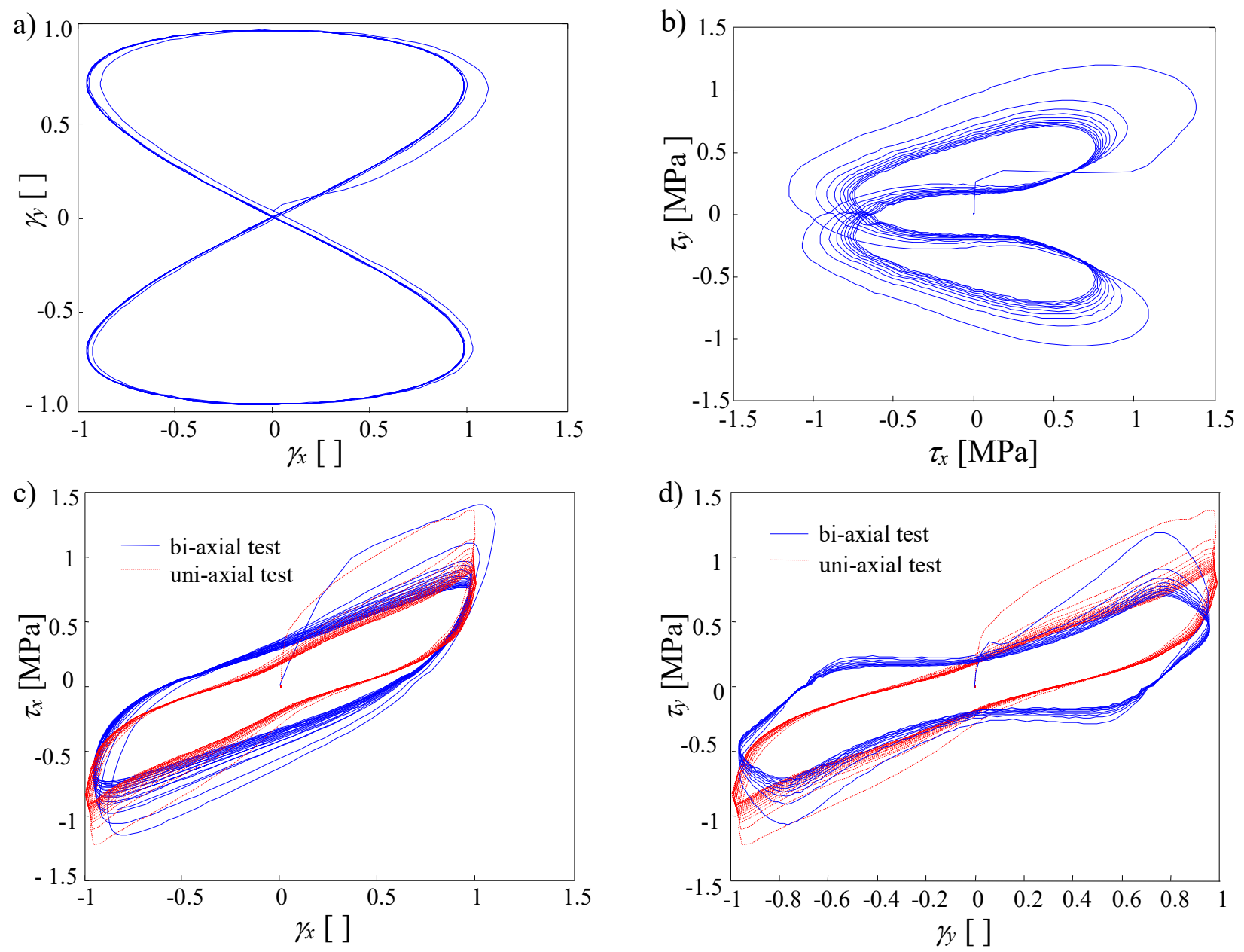

Figure 13. Biaxial tests: (a) figure 8-shaped strain trajectory at $100 \%$, (b) stress trajectory, (c) stress-strain response in the $x$-direction, (d) stress-strain response in the $y$-direction 
The stress-strain results in each orthogonal direction ( $x$ and $y)$ are again compared with those of the uni-axial double-shear test carried out at the same maximum strain amplitude (red dashed cycles in Figure $12 \mathrm{c}$ and $12 \mathrm{~d}$ ) and the increase in hysteresis over the uniaxial case is even more remarkable than in the case of the circular trajectory. In contrast, smaller differences are observed when the uniaxial data is compared to stress-strain results for either axis for the figure 8-shaped trajectory (Figure 13c and 13d), particularly in the $x$-direction, since coupling effects between responses along the $x$ and $y$ directions are reduced by the strain path passing at each cycle through the origin (corresponding to zero deformation).

From the results reported in this section the following useful remarks can be drawn: $(i)$ first of all a numerical model able to simulate the induced anisotropy of the Mullins effect is necessary to accurately simulate the seismic response of isolated structures, since in the first cycle of each test the maximum and minimum stresses in both the directions are very similar and only in the second cycle is a significant reduction of stress observed, (ii) coupling effects must also be simulated by the numerical model (eg 2D coupled plasticity models) because they may be of particular importance for both the transient and stable responses, depending on the strain trajectory shape during the seismic event.

\section{BIAXIAL CONSTITUTIVE MODEL FOR THE VIRGIN HDNR}

This section describes a biaxial shear constitutive model for HDNR accounting for the deformationinduced anisotropy observed in the experimental tests presented in the previous section. The proposed model introduces several advances in the description of the stress-softening behaviour of rubber over the models already available in the technical literature, which mainly focus on the stable horizontal biaxial behaviour, or describe the stress-softening by employing isotropic laws neglecting the anisotropic behaviour induced by the stress path. Based on the proposed material model, the force-displacement relationship of HDNR bearings used in seismic isolation can be evaluated through simple geometrical considerations. The validity of the assumption that the horizontal load-deflection behaviour of HDNR bearings can be estimated with sufficient accuracy from the shear stress-strain behaviour of the material follows if the deflection estimated from the bending compliance of the bearing is negligible compared to the sum of the shear compliances of all the rubber layers. For HDNR bearings used in seismic isolation (having large shape factors) this assumption is reasonable under the design condition, characterized by vertical loads and shear deformations less than limit values imposed by design codes [25]. It is worth to note that in these conditions the proposed model can be used both for circular and square bearings. Others situations where the axial force may influence significantly the horizontal bearing response, which becomes anisotropic for square bearings [13], are out of the scope of the present study.

\section{Proposed biaxial constitutive model}

Let $\boldsymbol{\gamma}=\left[\begin{array}{ll}\gamma_{x} & \gamma_{y}\end{array}\right]^{T}$ and $\boldsymbol{\tau}=\left[\begin{array}{ll}\tau_{x} & \tau_{y}\end{array}\right]^{T}$ denote respectively the vectors collecting the shear strains and shear stresses (in two orthogonal directions $x$ and $y$ ), as defined in the previous sections describing the experimental tests. The proposed model provides a relation between $\gamma$ and $\tau$. In particular, it is based on the additive decomposition of the stresses into two components. According to this concept, already employed in [2] for describing the one-dimensional response, the stress-strain material response is decomposed into two contributions:

$$
\boldsymbol{\tau}=\boldsymbol{\tau}_{0}+\boldsymbol{\tau}_{m}
$$

where the former $\left(\boldsymbol{\tau}_{0}\right)$ is the stable component not affected by the strain history, whereas the latter $\left(\boldsymbol{\tau}_{m}\right)$ describes the additional transient response, which degenerates as the stress-softening evolves during the strain history. The component $\tau_{0}$ of the stress is described by assuming a rheological 
model consisting of a nonlinear elastic spring $\left(\boldsymbol{\tau}_{e}\right)$, modelling the non linear elastic behaviour of the rubber and acting in parallel with other elements, modelling the dissipative component of the response. At least two elements are required to describe the dissipative behaviour of the HDNR: $\boldsymbol{\tau}_{v 1}$, representing the main dissipative contribution of the response, sensitive to very low strain rates but not to strain rates typical of seismic histories, and the term $\boldsymbol{\tau}_{v 2}$, which is a visco-elastic term sensitive to strain rates typical of seismic histories. The component $\tau_{0}$ may be expressed as follows:

$$
\tau_{0}=\tau_{e}(\gamma)+\tau_{v 1}\left(\gamma, \dot{\gamma}, \dot{\gamma}_{v 1}\right)+\tau_{v 2}\left(\gamma, \dot{\gamma}, \dot{\gamma}_{v 2}\right)
$$

The first term describing the elastic shear strain $\tau_{e}$ is oriented as the shear strain $\gamma$ and can be expressed as:

$$
\boldsymbol{\tau}_{e}=\left(a\|\gamma\|^{4}+b\|\gamma\|^{2}+c\right) \gamma
$$

where \|\|$\|$ denotes the norm of a vector. The main dissipative contribution $\boldsymbol{\tau}_{v 1}$ is modelled by a nonlinear plastic element in series with a viscous element (a simple Maxwell element), accounting for the long-term relaxation and providing the viscous shear strain rate

$$
\dot{\gamma}_{v 1}=v_{1} \tau_{v 1}
$$

The remaining part of the total strain is a plastic shear strain rate $\dot{\gamma}_{p}=\left(\dot{\gamma}-\dot{\gamma}_{v 1}\right)$ controlling the response of the plastic element. This contribution is described by a modified bounding surface (BS) model with vanishing elastic region [31]-[32], [4]-[5]. In the 2D shear stress space, the adopted boundary surface $R\left(\gamma_{p}\right)$ reduces to a curve with the expression

$$
R\left(\gamma_{p}\right)=\xi_{0}+\xi_{1}\left\|\gamma_{p}\right\|^{2}
$$

where the possible state of stress are limited by the condition $\left\|\boldsymbol{\tau}_{v 1}\right\| \leq R$. The assumed BS does not depend on the stress direction but it changes during the strain process, as $\gamma_{p}$ changes. The shear stress is provided by an incremental law and its progress is driven by the strain rate and by the position of the current stress with respect to the current BS. It is useful to define the current image stress $\hat{\boldsymbol{\tau}}_{v 1}=R\left(\gamma_{p}\right) \mathbf{n}$, where $\mathbf{n}=\dot{\gamma}_{p} /\left\|\dot{\gamma}_{p}\right\|$ is the unit vector in the direction of the plastic strain rate. The stress rate has the expression:

$$
\dot{\boldsymbol{\tau}}_{v 1}=\xi_{1} \dot{R} \mathbf{n}+\xi_{2}\left\|\dot{\gamma}_{p}\right\|\left(\hat{\boldsymbol{\tau}}_{v 1}-\boldsymbol{\tau}_{v 1}\right)
$$

The first term accounts for the variation of the BS and is proportional to the rate of $\left\|\gamma_{p}\right\|^{2}$, i.e., $\dot{R}=2 \gamma_{p} \cdot \dot{\gamma}_{p}$, the second stress contribution points to the image stress and is proportional to the distance between the current stress and the current image stress laying on the BS. According to the mono-dimensional case [2] the parameter $\xi_{2}$ depends linearly on the magnitude of the current plastic deformation:

$$
\xi_{2}=\xi_{2,1}+\xi_{2,2}\left\|\gamma_{p}\right\|
$$

It is worth noting note that, in accordance with experimental evidence, Eqn. (6) implies that the current stress is differently oriented with respect to the current total strain. Finally, a simple Maxwell element is sufficient to describe the viscous term $\boldsymbol{\tau}_{v 2}$ in the strain-rate range of interest, where a very low contribution is expected. The stress contribution is provided by the law: 


$$
\tau_{v 2}=E_{v 2}\left(\gamma-\gamma_{v 2}\right)
$$

where $\boldsymbol{\gamma}_{v 2}$ is the inelastic strain and $\boldsymbol{\tau}_{v 2}$ is the inelastic stress vectors oriented as the elastic strains $\left(\gamma-\gamma_{v 2}\right)$. The increment of the inelastic strain is along the same direction of the stress, i.e. of the elastic strain:

$$
\dot{\gamma}_{v 2}=v_{2} \tau_{v 2}
$$

The values of the model parameters characterizing the stable response are reported in Table 1 and are consistent with those employed for the one-dimensional constitutive law presented in [2] for the same rubber compound. The reader is referred to that paper for figures of the uniaxial simple shear data from which they were fitted and also for a more-in depth description of the model features and the differences with previous models employing the bounding surface concept [4],[5]. The assumption that this contribution to the stress is isotropic is made here and justified that the extended model fits the new biaxial data.

\begin{tabular}{|c|c|c|c|c|c|c|c|c|c|c|c|c|c|c|c|}
\hline \multicolumn{3}{|c|}{$\tau_{e}$} & \multicolumn{5}{|c|}{$\tau_{\nu 1}$} & \multicolumn{2}{|r|}{$\tau_{v 2}$} & \multicolumn{3}{|c|}{$\tau_{\mathrm{me}}$} & \multicolumn{3}{|c|}{$\tau_{\mathrm{mv}}$} \\
\hline $\mathrm{a}$ & $\mathrm{b}$ & $\mathrm{c}$ & $\xi_{0}$ & $\xi_{1}$ & $\xi_{2,1}$ & $\xi_{2,2}$ & $v_{1}$ & $E_{2}$ & $v_{2}$ & $\alpha_{e}$ & $\zeta_{\mathrm{e}}$ & $\beta$ & $\alpha_{v}$ & $\zeta_{\mathrm{v}}$ & $\beta$ \\
\hline $\mathrm{MPa}$ & $\mathrm{MPa}$ & $\mathrm{MPa}$ & $\mathrm{MPa}$ & $\mathrm{MPa}$ & - & - & $(\mathrm{MPa})^{-1} \mathrm{~s}^{-1}$ & $\mathrm{MPa}$ & $(\mathrm{MPa})^{-1} \mathrm{~s}^{-1}$ & - & - & - & - & - & - \\
\hline 0.015 & -0.05 & 0.28 & 0.14 & 0.08 & 3.5 & 1.5 & 0.4 & 0.068 & 8.5 & 1.7 & 0.25 & 0.4 & 2.2 & 0.13 & 0.4 \\
\hline
\end{tabular}

Table 1 - Model parameters of the stable (left) and transient (right) response

The extension of the transient response requires a different approach due to the deformationinduced anisotropy inherent to the Mullins effect. To model this direction-dependent material behaviour resort is made to the concept of representative directions [23], allowing the use of a one dimensional material law with damage parameters to simulate more complex $2 \mathrm{D}$ or $3 \mathrm{D}$ material behaviours. With reference to the 2D shear response, this homogenization technique is based on the selection of uniformly distributed directions in the plane and on the projection of the 2D shear strain state $\gamma$ to these directions. Let the unit vector $\mathbf{n}^{\theta}$ represent the direction corresponding to the angle $\theta$, the projection along that direction identifies the 1D (one-dimensional) strain:

$$
\varepsilon^{\theta}=\gamma \cdot \mathbf{n}^{\theta}
$$

For each direction, a 1D constitutive law relates the one-dimensional deformation measure $\varepsilon^{\theta}$ with a one-dimensional measure of the associated stress $\sigma^{\theta}$, oriented as $\mathbf{n}^{\theta}$. In addition, a set of internal variables is required to describe plastic and damage dissipative phenomena. These variables can be collected in the vector $\mathbf{v}^{\theta}$ as their values may differ from direction to direction. The response in the considered direction is known once the evolution laws of the internal variables are given:

$$
\dot{\mathbf{v}}^{\theta}=g\left(\varepsilon^{\theta}, \dot{\varepsilon}^{\theta} ; \mathbf{v}^{\theta}\right)
$$

and the stress is a derived quantity that can be expressed as follows

$$
\sigma^{\theta}=f\left(\varepsilon^{\theta}, \dot{\varepsilon}^{\theta} ; \mathbf{v}^{\theta}\right)
$$

If the selected directions are homogeneously and continuously distributed, the global 2D shearstress $\boldsymbol{\tau}_{m}$ due to the Mullins effect can be obtained by the following integral

$$
\boldsymbol{\tau}_{m}(\gamma, \dot{\gamma} ; \mathbf{v})=\frac{1}{\pi} \int_{o}^{\pi} f\left(\varepsilon^{\theta}, \dot{\varepsilon}^{\theta}, \mathbf{v}^{\theta}\right) \mathbf{n}^{\theta} d \theta
$$


where $\mathbf{v}$ contains the state variables for the different directions $\mathbf{v}^{\theta}$. The integral of Eqn. (13) is extended over $\pi$ rather than $2 \pi$ as is the case in [23], since the generic angle $\theta$ represents fibers along both the positive and negative direction.

In numerical applications, a discrete number of directions is assumed, thus the integral of Eqn. (13) can by approximated to a sum over all discrete directions and the state vector function reduces to a finite number of internal variables. In this paper, the response along each direction is the same as in [2] and accounts for the direction-dependent stress-softening. In particular, the transient response can be divided into two contributions:

$$
\sigma^{\theta}=\left(\tau_{m e}^{\theta}+\tau_{m v}^{\theta}\right) \mathbf{n}^{\theta}
$$

where the two damage contributions affecting the elastic and dissipative responses can be expressed as:

$$
\begin{gathered}
\tau_{m e}^{\theta}=\alpha_{e}\left(1-q_{e}^{\theta+}\right) \tau_{e}^{\theta} \text { for } \varepsilon^{\theta}>0 \\
\tau_{m e}^{\theta}=\alpha_{e}\left(1-q_{e}^{\theta-}\right) \tau_{e}^{\theta} \text { for } \varepsilon^{\theta}<0 \\
\tau_{m v}^{\theta}=\alpha_{v}\left(1-q_{v}^{\theta}\right)\left(\tau_{v 1}^{\theta}+\tau_{v 2}^{\theta}\right)
\end{gathered}
$$

As already assumed in [2] damage parameters $q_{e}^{\theta+}$ and $q_{e}^{\theta-}$ tend to limit values depending on the current value of the strain experienced along the corresponding direction. Their evolution laws, for $\varepsilon^{\theta}>0$, may be posed in the following form:

$$
\begin{array}{ll}
\dot{q}_{e}^{\theta+}=\zeta_{e} \mid \dot{\varepsilon}^{\theta-}\left(\left(\frac{\varepsilon^{\theta}}{\gamma_{\mathrm{mod}}}\right)^{\beta}-q_{e}^{\theta+}\right) & \text { if } q_{e}^{\theta+}<\left(\frac{\varepsilon^{\theta}}{\gamma_{\text {mod }}}\right)^{\beta} \\
\dot{q}_{e}^{\theta+}=0 & \text { if } q_{e}^{\theta+} \geq\left(\frac{\varepsilon^{\theta}}{\gamma_{\text {mod }}}\right)^{\beta}
\end{array}
$$

where $\gamma_{\text {mod }}$ is the maximum amplitude for which the model is deemed valid $\left(\gamma_{\text {mod }}=2.5\right.$ for this model) and $\zeta_{e}$ is a velocity parameter. Similarly for $\varepsilon^{\theta}<0$ the roles of $\dot{q}_{e}^{\theta+}$ and $\dot{q}_{e}^{\theta-}$ are interchanged in equations 16 and $\varepsilon^{\theta}$ is replaced by $\left|\varepsilon^{\theta}\right|$. For the damage parameter $q_{v}^{\theta}$ a similar evolution law is assumed, with the same maximum values, but with a different velocity parameter $\left(\zeta_{v}\right)$ and without the strain-direction dependency:

$$
\begin{array}{ll}
\dot{q}_{v}^{\theta}=\zeta_{v}\left|\dot{\gamma}^{\theta}\right|\left(\left(\frac{\left|\varepsilon^{\theta}\right|}{\gamma_{\text {mod }}}\right)^{\beta}-q_{v}^{\theta}\right) & \text { if } \quad q_{v}^{\theta}<\left(\frac{\left|\varepsilon^{\theta}\right|}{\gamma_{\text {mod }}}\right)^{\beta} \\
\dot{q}_{v}^{\theta}=0 & \text { if } q_{v}^{\theta} \geq\left(\frac{\left|\varepsilon^{\theta}\right|}{\gamma_{\text {mod }}}\right)^{\beta}
\end{array}
$$

The parameters characterizing the transient response $\left(\alpha_{e}, \zeta_{e}, \beta, \alpha_{v}, \zeta_{v}\right)$ are the same as those used in the mono-dimensional model described in [2] and they are reported in the right part of Table 1. Also for the contributions to the stable response in each direction $\left(\tau_{e}^{\theta}, \tau_{v 1}^{\theta}\right.$ and $\left.\tau_{v 2}^{\theta}\right)$, the same expressions of the mono-dimensional case are used. The values of the parameters for the response along 
direction $\theta$ need however to be scaled to obtain the same global stable uni-axial response of the uniaxial model. Table 2 reports the relation between the parameter adopted for the response along direction $\theta$ and the original mono-dimensional model.

Table 2 - Calibrated model parameters for the one-dimensional response in the $j$-th direction

\begin{tabular}{|c|c|c|c|c|c|}
\hline$\tau_{e}^{\theta}$ & \multicolumn{3}{|c|}{$\tau_{v 1}^{\theta}$} & \multicolumn{2}{|c|}{$\tau_{v 2}^{\theta}$} \\
\hline$a^{\theta}=3.2 a \quad b^{\theta}=2.8 b$ & $c^{\theta}=2 c$ & $\xi_{0}^{\theta}=\frac{\pi}{2} \xi_{0}$ & $E_{p}^{\theta}=2 E_{p}$ & $E_{v 2}^{\theta}=2 E_{v 2}$ & $v_{2}^{\theta}=v_{2} 0.5$ \\
\hline
\end{tabular}

It is worth noting that under the assumption of isotropic Mullins effect the model would simplify notably, and the approach based on representative directions would not be necessary to describe the transient response. In fact, under this assumption, the damage progresses with the norm of the displacement, as also assumed in [4]-[5], and Eq. 13-17 would be replaced by the following expressions:

$$
\boldsymbol{\tau}_{m}=\boldsymbol{\tau}_{m e}+\boldsymbol{\tau}_{m v}
$$

where

$$
\begin{gathered}
\boldsymbol{\tau}_{m e}=\alpha_{e}\left(1-q_{e}\right) \boldsymbol{\tau}_{e} \\
\boldsymbol{\tau}_{m v}=\alpha_{v}\left(1-q_{v}\right)\left(\boldsymbol{\tau}_{v 1}+\boldsymbol{\tau}_{v 2}\right)
\end{gathered}
$$

and the evolution laws for the both damage parameters $\left(q_{e}\right.$ and $q_{v}$, denoted in the following expressions as $q_{e / v}$ ) would be:

$$
\begin{aligned}
& \dot{q}_{e / v}=\zeta_{e / v}\|\dot{\gamma}\|\left(\left(\frac{\|\gamma\|}{\gamma_{\text {mod }}}\right)^{\beta}-q_{e / v}\right) \quad \text { if } \quad q_{e / v}<\left(\frac{\|\gamma\|}{\gamma_{\text {mod }}}\right)^{\beta} \\
& \dot{q}_{e / v}=0 \\
& \text { if } \quad q_{e / v} \geq\left(\frac{\|\gamma\|}{\gamma_{\bmod }}\right)^{\beta}
\end{aligned}
$$

By employing this approach the dependence of the Mullins effect on the strain direction and on the strain sign cannot be accounted for.

\section{Numerical simulation of shear tests}

In this paragraph the ability of the proposed model to simulate the transient and stable response under different shear strain paths is checked through the comparison between experimental tests and numerical simulations. Moreover, the importance of considering the deformation-induced anisotropy due to the Mullins effect is highlighted by showing the results of the simulations obtained by using the isotropic Mullins model with the same parameters as those used for the proposed model. Figure 14 reports the simulation of the cruciform tests. As expected, the proposed model is able to simulate that after cycling in one direction $(y)$ to reach relatively stable cycles (Figures $14 \mathrm{~b}$ and 15b), significant stress-softening still occurs in the orthogonal direction (Figures 14a), i.e., the response has not been fully stabilized as in the case of the isotropic Mullins effect (Figure 15a). 

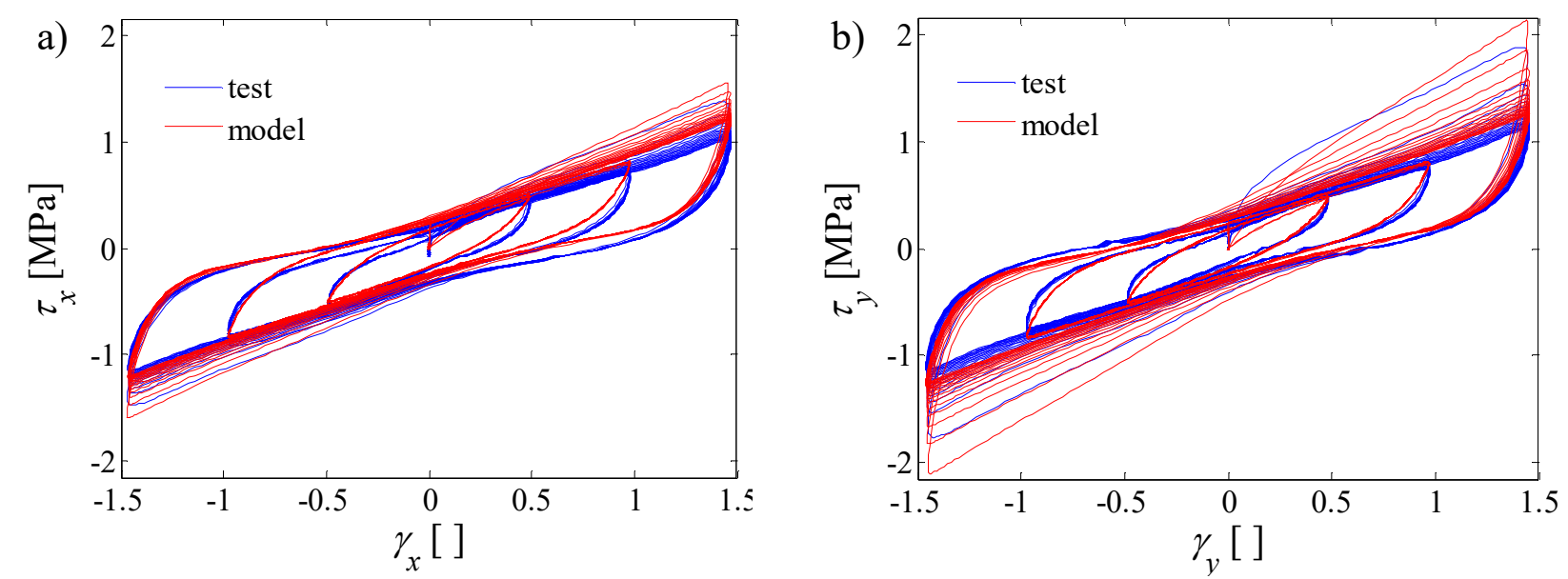

Figure 14. Numerical simulation of bi-axial shear tests with cruciform trajectory (proposed model).
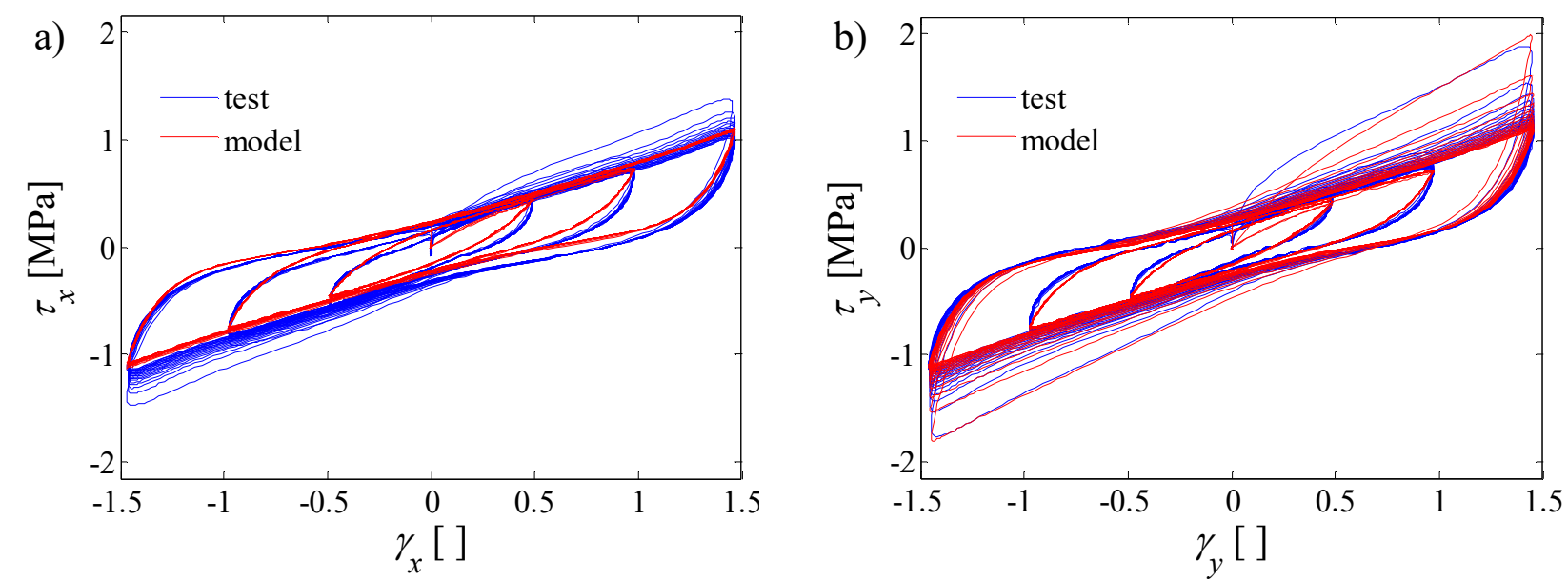

Figure 15. Numerical simulation of bi-axial shear tests with cruciform trajectory (isotropic model)

In Figure 16 the simulations of the circular tests are shown for both the cases of maximum amplitude $\gamma_{\max }=1.5$ (left column) and $\gamma_{\max }=1.0$ (right column). From the stress trajectory reported in Figures 16a and 16b it can be observed that the softening progresses similarly in the experimental and numerical responses. Also the stress-strain cycles in the two orthogonal directions $(x$ and $y)$ are very similar to each other for both the transient and the stable response.
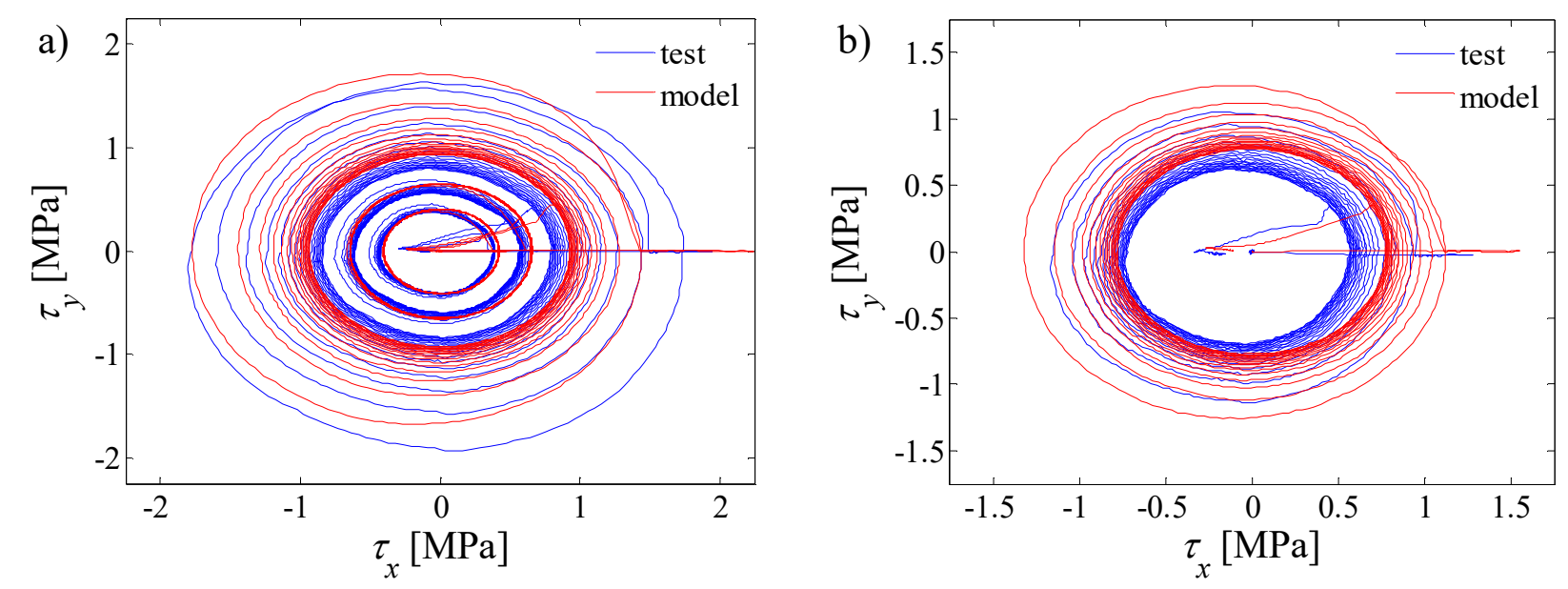

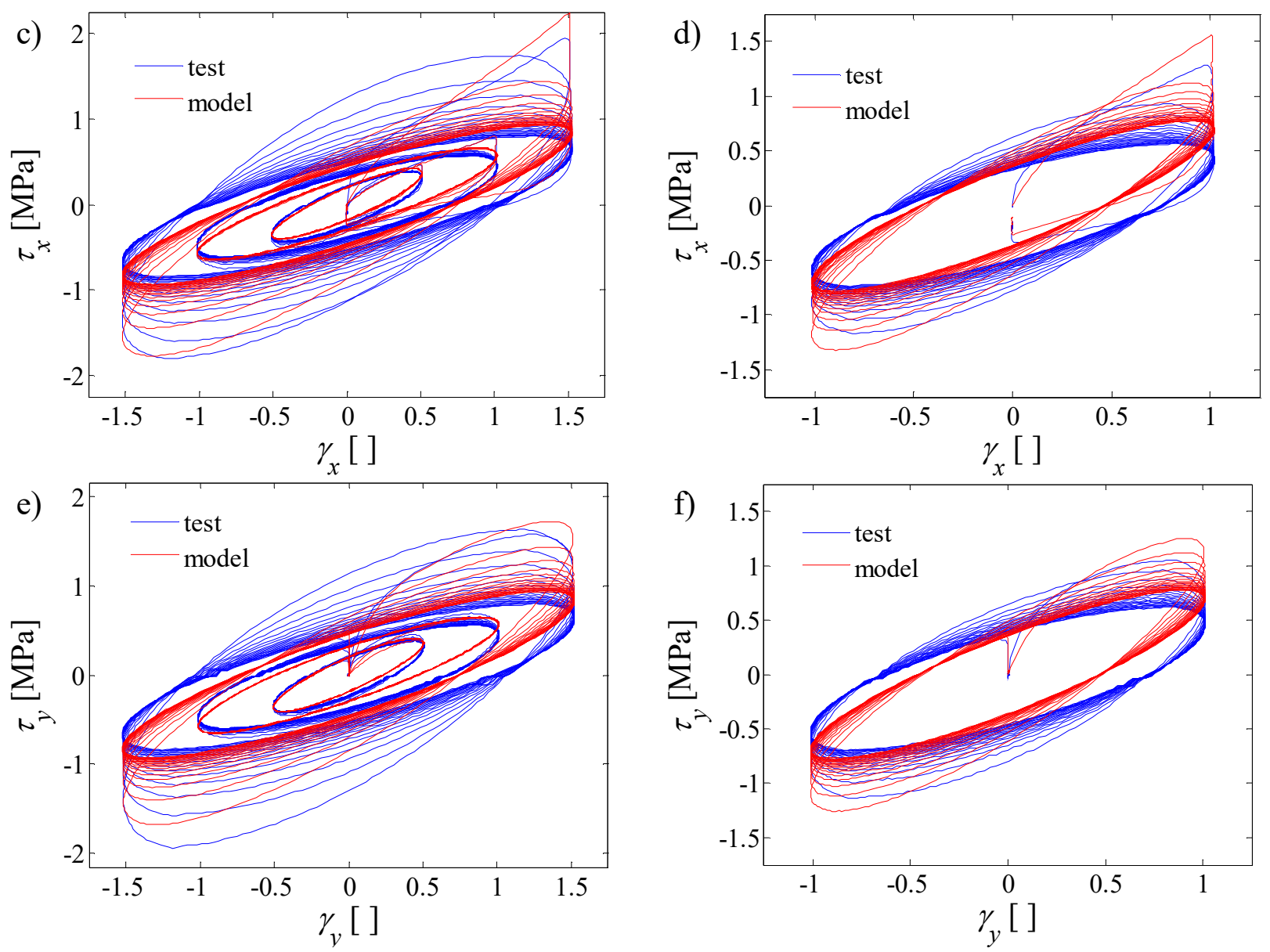

Figure 16. Numerical simulation of bi-axial shear tests with circular trajectory at maximum strain of (a, c, e) $150 \%$ and (b, d, f) $100 \%$.

For these tests, also the stress trajectory obtained by using the isotropic model are reported (Figure 17), revealing that in this case the softening progresses towards a stable state in significantly fewer cycles with respect to the experimental behaviour. This is again the consequence of the isotropic stress-softening assumption (i.e. softening in one direction has an effect in all the other directions). Finally, Figure 18 shows that the proposed model can accurately simulate also the experimental tests with square and figure- 8 trajectories.
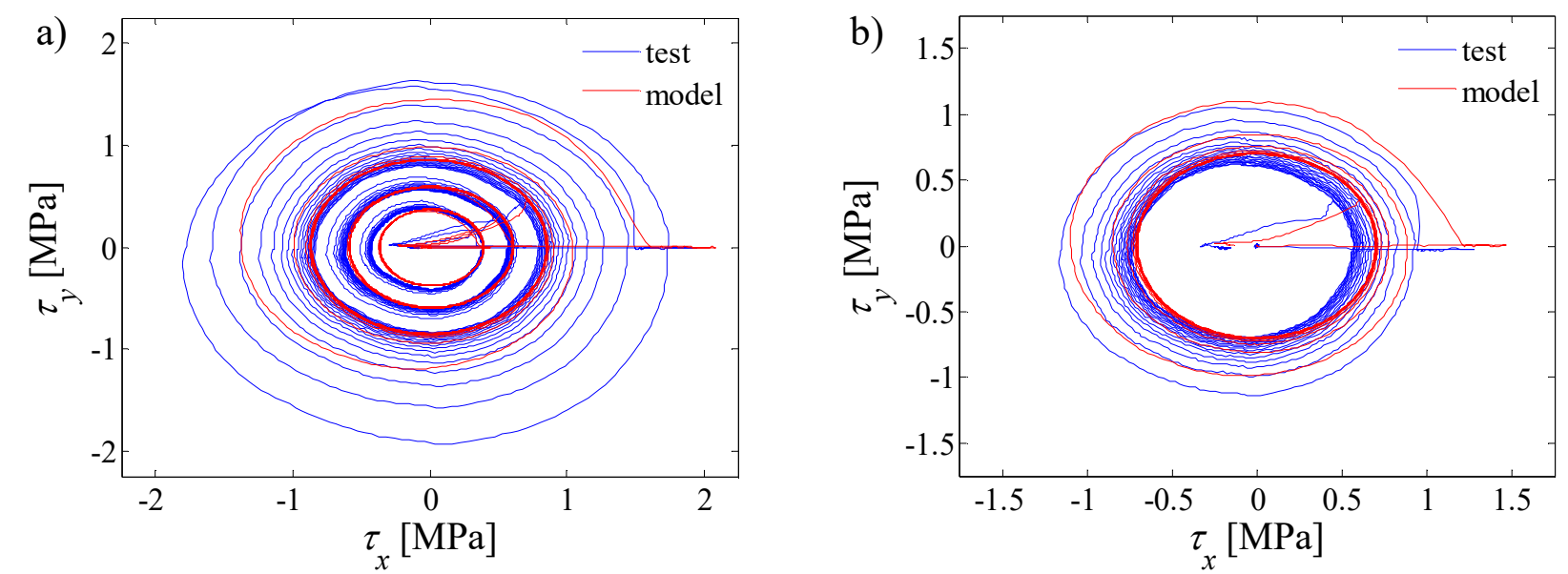

Figure 17. Numerical simulation of bi-axial shear tests with circular trajectories at maximum strain of (a) $150 \%$ and (b) $100 \%$ by using the isotropic model. 

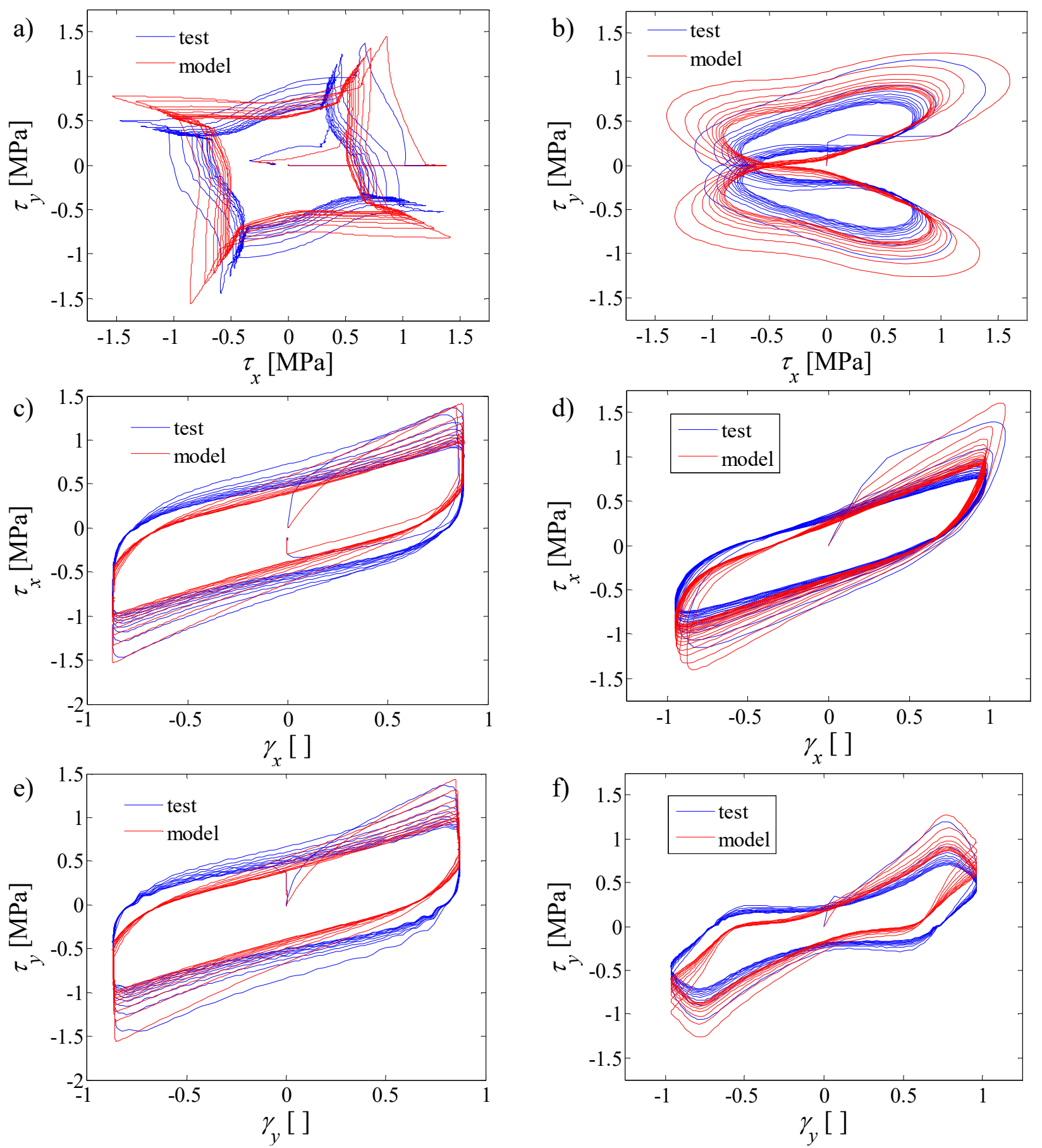

Figure 18. Numerical simulation of bi-axial shear tests with $(\mathrm{a}, \mathrm{c}, \mathrm{e})$ square and $(\mathrm{b}, \mathrm{d}, \mathrm{f})$ figure 8-shaped trajectories at maximum strain of $100 \%$.

It is worth noting that the proposed model is able to simulate (by using the same parameters) both bi-axial and uni-axial responses. In order to show this aspect, the simulation of the uni-axial double shear tests reported in Figures 2 and 4 are illustrated in Figure 19. In particular, in Figure 19a the symmetric cyclic test of Figure 2 at $\gamma_{\max }=2.0$ is simulated, whereas Figure $19 \mathrm{~b}$ reports the simulation of the asymmetric test of Figure 4. These plots confirm the ability of the model to account for the direction dependence of the Mullins effect also for the uni-axial case. 

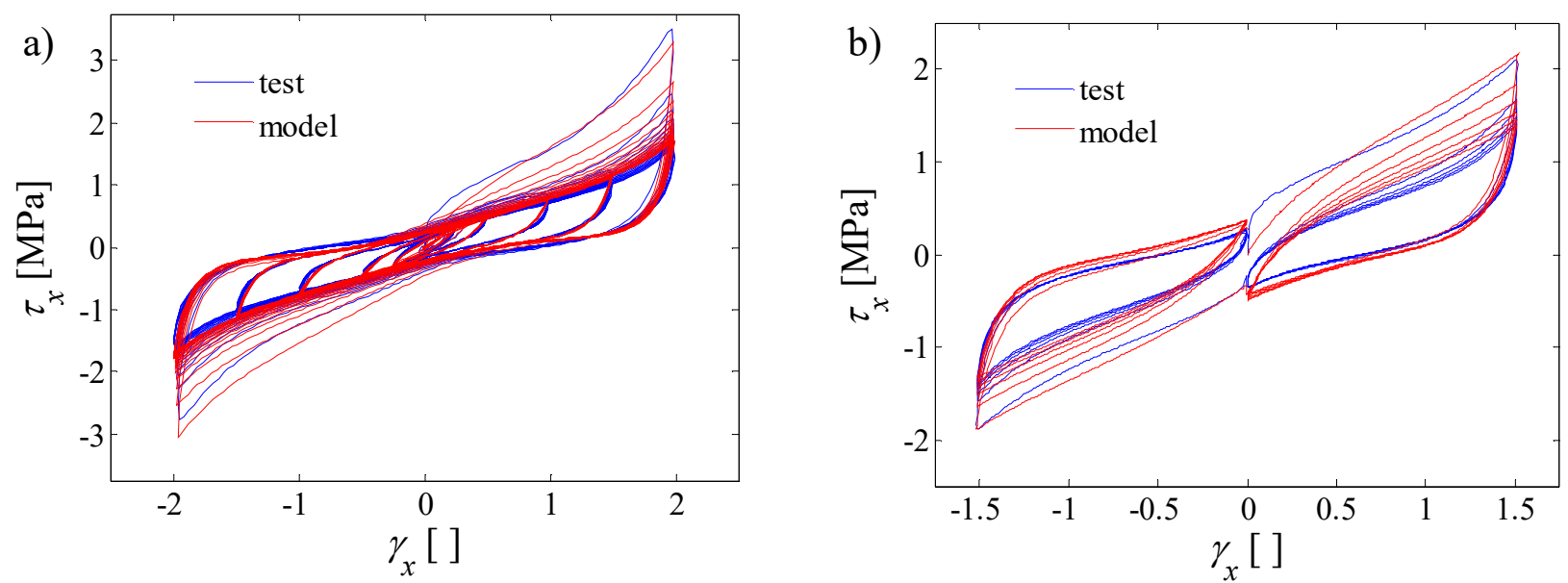

Figure 19. Numerical simulation of uni-axial shear tests of (a) Figure 2a and (b) Figure 4

\section{CONCLUSIONS}

This study illustrates the results of an extensive experimental campaign aimed at characterizing the complex stress-softening behaviour of high damping natural rubber (HDNR) bearings, commonly employed in seismic isolation. A series of uni-axial double shear tests, rotated double shear tests, and biaxial test has been performed on separate virgin HDNR samples showing two important features of the stress-strain behaviour: $(i)$ the softening due to the Mullins effect is directiondependent and so will result in anisotropic mechanical properties of rubber if the scragging is not isotropic (ii) there is coupling between the responses in the two shear directions, that is, the stress response along one axis depends not only on the coaxial strain, but also on details of the strain in the orthogonal axis. These effects need to be considered when simulating the seismic response of structural systems isolated by HDNR bearings, since they may affect significantly both the transient and stable responses depending on the deformation path.

A numerical biaxial model able to accurately describe these significant features of the HDNR bearing behaviour is proposed, based on the additive decomposition of the stresses. The stable behaviour is described by a 2D nonlinear elastic model in parallel with a 2D plasticity model (for the hysteretic contribution) and a 2D Maxwell model (for the viscous contribution). On the other hand, the transient response, characterized by the deformation-induced anisotropy inherent to the Mullins effect, is described by resorting to the concept of representative directions. This approach involves a homogenization of the responses of a set of uniformly distributed directions in the plane, each characterized by the one dimensional material law (with damage parameters) already developed for the one-dimensional case.

The effectiveness of the proposed model is verified by comparing the results of both the uni-axial and the bi-axial shear tests with the corresponding numerical simulations. Based on simple considerations of the isolator geometry, the model can be employed to accurately simulate the horizontal biaxial response of virgin HDNR bearings or of HDNR bearings subjected to a long period of rest, such that the virgin properties can be assumed to have fully recovered. Dynamic analyses accounting for the induced-anisotropy of the Mullins effects may be carried out to properly evaluate the seismic reliability of structures isolated by HDNR bearings under design actions. The proposed model could also be used to develop a 3D model of virgin HDNR bearings able to capture all the biaxial phenomena presented here, along with coupling effects between the horizontal and vertical behaviour, which could be useful to describe the cases where axial force fluctuations influence significantly the horizontal bearing response.

\section{REFERENCES}

[1] Mullins L. Softening of rubber by deformation. Rubber Chemistry and Technology 1969; 42(1): 339-362.

[2] Tubaldi E, Ragni L, Dall'Asta A, Ahmadi H, Muhr A. Stress-softening behaviour of HDNR bearings: modelling and influence on the seismic response of isolated structures. Earthquake Engineering And Structural Dynamics 2017; Published online in Wiley Online Library (wileyonlinelibrary.com). DOI: 10.1002/eqe.2897. 
[3] Huang W-H. Bi-Directional Testing, Modeling, and System Response of Seismically Isolated Bridges. Ph. D. thesis, University of California, Berkeley; 2002.

[4] Grant DN, Fenves GL, Whittaker AS. Bidirectional modeling of high-damping rubber bearings. Journal of Earthquake Engineering 2004; 8(1):161-185.

[5] Grant DN, Fenves GL, Auricchio F. Modelling and analysis of High-damping Rubber Bearings for the seismic protection of bridges. Iuss Press, Pavia; 2005.

[6] Clark, P., I. D. Aiken, and J. M. Kelly. Experimental studies of the ultimate behavior of seismically-isolated structures. Technical Report UCB/EERC\{97/18, Earthquake Engineering Research Center, University of California, Berkeley; 1997.

[7] Kumar M. Seismic isolation of nuclear power plants using elastomeric bearings. PhD Dissertation, Departement of Civil, Structural and Environmental Engineering, University at Buffalo; 2015.

[8] McKenna F, Fenves GL, Scott MH. OpenSees: open system for earthquake engineering simulation. Pacific Earthquake Engineering Center, University of California, Berkeley, CA; 2006.

[9] Tubaldi E, Mitoulis SA, Ahmadi H, Muhr A. A parametric study on the axial behaviour of elastomeric isolators in multi-span bridges subjected to horizontal seismic excitations. Bulletin of Earthquake Engineering 2016; 14(4): $1285-1310$

[10] Abe M, Yoshida J, Fujino Y. Multiaxial Behaviours of Laminated Rubber Bearings and their Modeling. I: Experimental Study, Journal of Structural Engineering 2005;130(8): 1119-1132.

[11] Abe M, Yoshida J, Fujino Y. Multiaxial Behaviours of Laminated Rubber Bearings and their Modeling. II: Modelling, Journal of Structural Engineering 2004;130(8):1133-1144.

[12] Yamamoto S, Kikuchi M, Ueda1 M, Aiken IA. A mechanical model for elastomeric seismic isolation bearings including the influence of axial load. Earthquake Engineering And Structural Dynamics 2009; 38:157-180.

[13] Kikuchi M, Nakamura T, Aiken ID. Three-dimensional analysis for square seismic isolation bearings under large shear deformations and high axial loads. Earthquake Engineering and Structural Dynamics 2010; 39:1513-1531.

[14] Kato H. et al. Analytical Model for Elastoplastic and Creep-Like Behavior of High-Damping Rubber Bearings, Journal of Structural Engineering 2014; 141(9): http://dx.doi.org/10.1061/(ASCE)ST.1943-541X.0001181.

[15] Govindjee S, Simo J. A micro-mechanically based continuum damage model for carbon black-filled rubbers incorporating Mullins' effect. Journal of the Mechanics and Physics of Solids 1991; 39(1): 87-112.

[16] Dorfmann A, Ogden RW. A pseudo-elastic model for loading, partial unloading and reloading of particlereinforced rubber. International Journal of Solids and Structures 2003; 40(2): 699-714.

[17] Govindjee S, Simo J. Mullins effect and the strain amplitude dependence of the storage modulus. International Journal of Solids and Structures 1992; 29(14-15):1737-1751.

[18] Besdo D, Ihlemann J, Kingston J, Muhr A. Modelling inelastic stress-strain phenomena and a scheme for efficient experimental characterization. Constitutive Models for Rubber III, 309-317. Swets \& Zeitlinger, Lisse; 2003.

[19] Diercks N, Lion A. Modeling deformation-induced anisotropy using 1D-laws for Mullins-Effect. Constitutive models for rubber VIII, 419-424, Taylor \& Francis Group, London; 2013.

[20] Dorfmann A, Pancheri FQ. A constitutive model for the Mullins effect with changes in material symmetry. International Journal of Nonlinear Mechanics 2012; 47:874-887.

[21] Wulf H, Ihlemann J. Simulation of self-organization processes in filled rubber and their influence on anisotropic Mullins effect. Constitutive models for rubber VIII, Taylor \& Francis Group, London 2013, pp.425-430.

[22] Göktepe S., Miehe C. A micro-macro approach to rubber-like materials. Part III: The micro-sphere model of anisotropic Mullins-type damage. Journal of the Mechanics and Physics of Solids 2005; 53:2259-2283

[23] Freund M, Ihlemann J. Generalization of one-dimensional material models for the finite element method. ZAMM - Z. Angew. Math. Mech. 2010; 90 (5): $399-417$.

[24] Kelly JM, Konstantinidis D. Mechanics of Rubber Bearings for Seismic and Vibration Isolation. John Wiley \& Sons, Ltd; 2011.

[25] European Committee for Standardization (ECS). EN 15129, Anti-seismic devices, CEN, Bruxelles; 2009.

[26] Burtscher SL, Dorfmann A. Compression and shear tests of anisotropic high damping rubber bearings. Engineering Structures 2004; 26:1979-1991

[27] Dall'Asta A, Ragni L. Experimental Tests and Analytical Model of High Damping Rubber Dissipating Devices, Engineering Structures 2006; 28(13):1874-1884.

[28] Dall'Asta A, Ragni L. Nonlinear behavior of dynamic systems with high damping rubber devices, Engineering Structures 2008; 30 (12):3610-3618.

[29] Dall'Asta A, Ragni L. Dynamic systems with high damping rubber: Nonlinear behaviour and linear approximation, Earthquake Engineering and Structural Dynamics 2008; 37(13):1511-2526.

[30] International Organization for Standardization (ISO). ISO 4664-1:2011 rubber, vulcanized or thermoplastic determination of dynamic properties - Part 1: general guidance, Geneva; 2011.

[31] Dafalias Y, Popov E. A model for nonlinearly hardening materials for complex loading. Acta Mechanica 1975; 21 (3): 173-192.

[32] Österlöf R., Wentzel H., Kari L., Diercks N., Wollscheid D. Constitutive modeling of the amplitude and frequency dependency of filled elastomers utilizing a modified Boundary Surface Model. International Journal of Solids and Structures 2014; 51: 3431-3438. 